\title{
Hopf bifurcation and the existence and stability of closed orbits in three-sector models of optimal endogenous growth
}

\author{
${ }^{1}$ Research Institute for Economics and Business Administration, Kobe University, Kobe, Japan \\ ${ }^{2}$ Institute of Economic Research, Kyoto University, Kyoto, Japan, E-mail: sigoka@kier.kyoto-u.ac.jp
}

\begin{abstract}
:
The present paper constructs a family of three-sector models of optimal endogenous growth, and conducts exact bifurcation analysis. In so doing, original six-dimensional equilibrium dynamics is decomposed into fivedimensional stationary autonomous dynamics and one-dimensional endogenously growing component. It is shown that the stationary dynamics thus decomposed undergoes supercritical Hopf bifurcation. It is inferred from the convex structure of our model that the dimension of a stable manifold of each closed orbit thus bifurcated in this five-dimensional dynamics should be two.
\end{abstract}

Keywords: Hopf bifurcation, multi-sector model, optimal endogenous growth model

DOI: 10.1515/snde-2019-0017

\section{Introduction}

The present paper constructs a continuous-time model of optimal endogenous growth in which an optimal path fluctuates around a balanced growth path (i.e. BGP). The model extends the two-sector models of Uzawa (1965) and Caballé and Santos (1993), in which an optimal path do not fluctuate around the BGP. The main result is to show that this would no longer be the case if the third stock variable is included in the model. By using a bifurcation theorem, the paper demonstrates that, with three stock variables, economic fluctuations may occur along an optimal path around the BGP.

Uzawa (1965) introduces a continuous time and two-sector model of optimal endogenous growth with physical and human capitals and with a linear felicity function. Owing to the linearity of felicity function, transitional dynamics in his model exhibits corner solutions. Caballé and Santos (1993) construct a large class of continuous time and two-sector models of optimal endogenous growth with physical and human capitals and with a strictly concave felicity function. They use convex technology that is more general than that used by Uzawa (1965). Owing to the strict concavity of felicity function, transitional dynamics in their model exhibits robust interior solutions. They first consider the class of technologies such that an educational sector uses human capital alone as an input of capital stock, and they show that within this class, if an optimal BGP exists, it is unique and globally asymptotically stable. They also consider the class of technologies such that an educational sector uses both physical and human capitals as an input of capital stock, and they treat some problems on interior transitional dynamics. From a purely logical point of view, one could not exclude the possibility that BGP might lose either uniqueness or stability as technology would vary within this class. However, even if the BGP might lose stability within the class, there would be no interior endogenous fluctuations around the BGP, as discussed in the next paragraph.

Consider a continuous time and multi-sector model of optimal endogenous growth that includes two heterogenous capitals with a strictly concave felicity function and with convex technology. And suppose that structures of preference and of technology in this hypothetical model permit a BGP to exist. The convex structure of this model implies that if an interior optimal solution would exist, it should be unique. And equilibrium dynamics of an interior optimal path in the model could be described by four-dimensional autonomous differential equation that is composed of two heterogenous capitals and of two imputed prices of these two capitals. Thus the original four-dimensional dynamics includes two predetermined and two non-predetermined variables. And by means of some log-linear variable transformations, this four-dimensional autonomous dynamics should be able to be decomposed into three-dimensional stationary autonomous dynamics and onedimensional endogenously growing component in such a way that the resulting three-dimensional stationary 
dynamics includes one predetermined and two non-predetermined variables and that a steady state of this stationary dynamics corresponds to the BGP of the original four-dimensional dynamics. Suppose that a steady state of the stationary dynamics is hyperbolic. Since an interior equilibrium is at most unique, and since the stationary dynamics includes only one predetermined variable, the hyperbolic steady state should have at least two unstable roots, and only one characteristic root of it could change the sign of its real part. ${ }^{1}$ If interior equilibrium should be at most one, (in other words, if equilibrium is determinate,) and if one would work within a continuous time model, one should have at least two predetermined variables in stationary dynamics in order to obtain endogenous fluctuations such as due to a stable closed orbit. As long as one works within the framework of our hypothetical continuous time and multisector model of optimal endogenous growth with two heterogenous capitals, one could not obtain endogenous fluctuations around the BGP.

This thought experiment suggests two methods of obtaining endogenous fluctuations around a BGP in continuous time and multisector models of endogenous growth with heterogenous capitals. As one method, one might increase the number of heterogenous capitals in order to make the resulting stationary dynamics include more than two predetermined variables, while keeping the number of interior equilibrium at most one. As an alternative method, one might introduce external effects into the above hypothetical model in order to make equilibrium indeterminate, while keeping the number of capital two. The present study pursues the first approach, because this approach has not yet been pursued in the literature, ${ }^{2}$ and because in sharp contrast, the second approach has already been pursued extensively in the literature. ${ }^{3}$

We construct a family of multisector models of optimal endogenous growth with three heterogenous capitals and with a strictly concave felicity function, and conduct exact bifurcation analysis. In so doing, original six-dimensional equilibrium dynamics is decomposed into five-dimensional stationary autonomous dynamics and one-dimensional endogenously growing component. Fundamental characteristics compatible with the existence of a BGP, combined with the choice of a Cobb-Douglas technology, introduce strong log-linear structure into our model, which in turn enables us to elicit two-dimensional autonomous stationary dynamics from the five-dimensional stationary dynamics under appropriate variable transformations. The present study shows that the two-dimensional stationary dynamics thus elicited undergoes supercritical Hopf bifurcation. The convex structure of our model implies that if interior equilibrium would exist, it should be unique, which implies in turn that the dimension of a stable manifold of each closed orbit thus bifurcated in the five-dimensional stationary system should be two, since the bifurcation is supercritical and since the number of predetermined variable in this system is two. In other words, a closed orbit around the BGP is locally determinate and stable.

The rest of the paper is composed of the following sections. Section 2 presents our model. Section 3 characterizes equilibrium dynamics of the model. Section 4 applies bifurcation analyses to the equilibrium dynamics. A set of appendices has been gathered at the end of the paper.

\section{The model}

The present study considers a continuous time and three-sector model of optimal endogenous growth with three types of heterogenous capital goods $K_{i}, i=1,2,3$. Each sector accumulates each type of capital goods. The goods produced by the first sector is also utilized as consumption goods $C$. Formally the model is given by the following intertemporal optimization problem.

$$
\operatorname{Max}_{C, K_{i j}, i, j=1,2,3} \int_{0}^{\infty} \frac{C^{1-\sigma}-1}{1-\sigma} e^{-\rho t} d t
$$

subject to

$$
\begin{aligned}
& C>0, K_{i j} \geq 0, i, j=1,2,3, K_{i}>0, i=1,2,3, \\
& \dot{K}_{1}=e_{1}\left(K_{11}\right)^{\beta_{11}}\left(K_{21}\right)^{\beta_{21}}\left(K_{31}\right)^{\beta_{31}}-C-g K_{1} \\
& \dot{K}_{2}=e_{2}\left(K_{12}\right)^{\beta_{12}}\left(K_{22}\right)^{\beta_{22}}\left(K_{32}\right)^{\beta_{32}}-g K_{2} \\
& \dot{K}_{3}=e_{3}\left(K_{13}\right)^{\beta_{13}}\left(K_{23}\right)^{\beta_{23}}\left(K_{33}\right)^{\beta_{33}}-g K_{3} \\
& K_{11}+K_{12}+K_{13}=K_{1} \\
& K_{21}+K_{22}+K_{23}=K_{2} \\
& K_{31}+K_{32}+K_{33}=K_{3} \\
& K_{1}(0)=\bar{K}_{1}>0, K_{2}(0)=\bar{K}_{2}>0, K_{3}(0)=\bar{K}_{3}>0,
\end{aligned}
$$


where $K_{i j}$ is an input of the $i$-th capital into the $j$-th sector, and $\bar{K}_{i}>0$ is an initial endowment of the $i$-th capital, and where $\sigma>0, \rho>0, e_{i}>0, \beta_{i j}>0$, and $g \geq 0$. We assume constant returns to scale technology.

$$
\beta_{1 j}+\beta_{2 j}+\beta_{3 j}=1, j=1,2,3 .
$$

For the given intertemporal optimization problem (1), $C$, and $K_{i j}, i, j=1,2,3$, are control variables, $K_{i}, i=1,2,3$, are state variables, and $\left(K_{1}(0), K_{2}(0), K_{3}(0)\right)=\left(\bar{K}_{1}, \bar{K}_{2}, \bar{K}_{3}\right)$ is an initial condition. An optimal solution of the problem (1) should satisfy

$$
\int_{0}^{\infty}\left|\frac{C^{1-\sigma}-1}{1-\sigma}\right| e^{-\rho t} d t<+\infty .
$$

The condition (2) is called the summability condition.

Intertemporal elasticity of substitution $1 / \sigma$ and rate of time preference $\rho$ are constant, and production functions are homogenous of degree one with respect to reproducible production factors. The structures of preference and of technology are compatible with the existence of a BGP. The present study imposes further restrictions on parameters characterizing preference and technology in order to guarantee that there is an interior optimal BGP on which consumption goods and each capital grows endogenously with positive constant rates and on which the transversality and the summability conditions are satisfied.

Let $b_{i}, i=1,2,3$, be defined as

$$
\begin{aligned}
& b_{1}:=\left(\beta_{11}\right)^{\beta_{11}}\left(\beta_{21}\right)^{\beta_{21}\left(\beta_{31}\right)^{\beta_{31}}} \\
& b_{2}:=\left(\beta_{12}\right)^{\beta_{12}}\left(\beta_{22}\right)^{\beta_{22}}\left(\beta_{32}\right)^{\beta_{32}} \\
& b_{3}:=\left(\beta_{13}\right)^{\beta_{13}}\left(\beta_{23}\right)^{\beta_{23}}\left(\beta_{33}\right)^{\beta_{33}} \text {. }
\end{aligned}
$$

Let $\mathbf{B}$ be a $3 \times 3$ matrix defined as

$$
\mathbf{B}:=\left[\begin{array}{lll}
\beta_{11} & \beta_{12} & \beta_{13} \\
\beta_{21} & \beta_{22} & \beta_{23} \\
\beta_{31} & \beta_{32} & \beta_{33}
\end{array}\right] .
$$

In the present study we make the following assumption.

\section{Assumption 1}

1. There is a positive constant $\mu>0$ that satisfies the following conditions.

a. $e_{1}=\frac{\rho+g+\sigma \mu}{b_{1}}, e_{2}=\frac{\rho+g+\sigma \mu}{b_{2}}$, and $e_{3}=\frac{\rho+g+\sigma \mu}{b_{3}}$.

b. $\rho-(1-\sigma) \mu>0$.

2. $\operatorname{det} \mathbf{B} \neq 0$.

Let $\omega>0$ be defined as

$$
\omega:=\rho+g+\sigma \mu
$$

Let $\mathbf{C}$ denote the inverse matrix of $\mathbf{B}$.

$$
\mathbf{C}:=\mathbf{B}^{-1}
$$

Let $1:=(1,1,1)$. Then we have

$$
1 B=1 \quad 1 C=1
$$

Let $\mathbf{I}_{3}$ denote the $3 \times 3$ identity matrix, and let $\mathbf{e}_{1}:=(1,0,0)^{\mathrm{T}}{ }^{4}$ Then we have the following result. See Appendix 1 for the proof. 


\section{Lemma 1}

Suppose that Assumption 1 is satisfied.

1. $\operatorname{det}\left(\omega \mathbf{C}-(g+\mu) \mathbf{I}_{3}\right) \neq 0$.

2. Each element of the $3 \times 1$ vector $\left(\omega \mathbf{C}-(g+\mu) \mathbf{I}_{3}\right)^{-1} \mathbf{e}_{1}$ is strictly positive.

Lemma 1 will guarantee the existence of an interior BGP. If one provides $\rho, \sigma, \mu, g$, and $\mathbf{B}$ with numeric values, then numeric values of $e_{i}, i=1,2,3, \mathbf{C}, \omega$, and $\left(\omega \mathbf{C}-(g+\mu) \mathbf{I}_{3}\right)^{-1} \mathbf{e}_{1}$ are uniquely determined. The following three parametric examples satisfy Assumption 1.

\section{Example 1}

$$
\rho=\frac{5}{100}, \sigma=\frac{1}{4}, \mu=\frac{3}{100}, g=\frac{2}{100} \text {, and }
$$

$$
\mathbf{B}=\left[\begin{array}{ccc}
\frac{7}{15} & \frac{1}{5} & \frac{1}{3} \\
\frac{1}{5} & \frac{11}{20} & \frac{1}{4} \\
\frac{1}{3} & \frac{1}{4} & \frac{5}{12}
\end{array}\right]
$$

\section{Example 2}

$$
\rho=\frac{5}{100}, \sigma=\frac{3}{2}, \mu=\frac{3}{100}, g=\frac{2}{100} \text {, and }
$$

$$
\mathbf{B}=\left[\begin{array}{ccc}
\frac{5}{12} & \frac{1}{4} & \frac{1}{3} \\
\frac{1}{4} & \frac{11}{20} & \frac{1}{5} \\
\frac{1}{3} & \frac{1}{5} & \frac{7}{15}
\end{array}\right]
$$

\section{Example 3}

$$
\rho=\frac{5}{100}, \sigma=\frac{5}{100}, \mu=\frac{3}{100}, g=\frac{2}{100} \text {, and }
$$

$$
\mathbf{B}=\left[\begin{array}{ccc}
\frac{1}{6} & \frac{1}{3} & \frac{1}{2} \\
\frac{1}{3} & \frac{5}{12} & \frac{1}{4} \\
\frac{1}{2} & \frac{1}{4} & \frac{1}{4}
\end{array}\right] .
$$

\section{Equilibrium dynamics}

\subsection{Maximum principle}

In the present study that treats a multi-sector model, we have to make explicit whether a given vector is either a row vector or a column vector, in order to avoid possible confusion. In the present study, $\mathbb{R}^{n}$ refers to a set of all real $1 \times n$ row vectors. Hence if $\mathbf{a} \in \mathbb{R}^{n}$, then a refers to a $1 \times n$ row vector, and $\mathbf{a}^{\mathrm{T}}$ refers to an $n \times 1$ column vector.

The problem (1) is solved by defining the current value Hamiltonian $\mathcal{H}$ and the current value maximized Hamiltonian $\mathcal{H}^{*}$, and by applying the maximum principle to $\mathcal{H}^{*}$. Let $\mathbf{K}=\left(K_{1}, K_{2}, K_{3}\right) \in \mathbb{R}_{++}^{3}, \mathbf{P}=\left(P_{1}, P_{2}, P_{3}\right) \in$ $\mathbb{R}_{++}^{3}$, and $\mathbf{W}=\left(W_{1}, W_{2}, W_{3}\right) \in \mathbb{R}_{+}^{3}$. The current value Hamiltonian $\mathscr{H}=\mathscr{H}\left(\mathbf{K}, \mathbf{P}, C,\left\{K_{i j}\right\}_{i, j=1,2,3}, \mathbf{W}\right)$ is given by

$$
\begin{aligned}
\mathscr{H}= & \frac{C^{1-\sigma}-1}{1-\sigma} \\
& +P_{1}\left(e_{1}\left(K_{11}\right)^{\beta_{11}}\left(K_{21}\right)^{\beta_{21}}\left(K_{31}\right)^{\beta_{31}}-C-g K_{1}\right) \\
& +P_{2}\left(e_{2}\left(K_{12}\right)^{\beta_{12}}\left(K_{22}\right)^{\beta_{22}}\left(K_{32}\right)^{\beta_{32}}-g K_{2}\right) \\
& +P_{3}\left(e_{3}\left(K_{13}\right)^{\beta_{13}}\left(K_{23}\right)^{\beta_{23}}\left(K_{33}\right)^{\beta_{33}}-g K_{3}\right) \\
& +W_{1}\left(K_{1}-\left(K_{11}+K_{12}+K_{13}\right)\right) \\
& +W_{2}\left(K_{2}-\left(K_{21}+K_{22}+K_{23}\right)\right) \\
& +W_{3}\left(K_{3}-\left(K_{31}+K_{32}+K_{33}\right)\right),
\end{aligned}
$$

where $C>0$, and $K_{i j} \geq 0, i, j=1,2,3$, and where $P_{i}$ is an imputed price of $K_{i}$, and $W_{i}$ is a rental price of $K_{i}$. Note that $\mathscr{H}=\mathscr{H}\left(\mathbf{K}, \mathbf{P}, C,\left\{K_{i j}\right\}_{i, j=1,2,3}, \mathbf{W}\right)$ is concave in $\left(\mathbf{K}, C,\left\{K_{i j}\right\}_{i, j=1,2,3}\right)$. 
Let $c_{i j}$ denote the $(i, j)$-element of $\mathbf{C}$ for each $i, j=1,2,3$. Let $W_{i}(\mathbf{P}), i=1,2,3$ be functions of $\mathbf{P} \in \mathbb{R}_{++}^{3}$ defined as

$$
\begin{aligned}
& W_{1}(\mathbf{P}):=\omega P_{1}^{c_{11}} P_{2}^{c_{21}} P_{3}^{c_{31}} \\
& W_{2}(\mathbf{P}):=\omega P_{1}^{c_{12}} P_{2}^{c_{22}} P_{3}^{c_{32}} \\
& W_{3}(\mathbf{P}):=\omega P_{1}^{c_{13}} P_{2}^{c_{23}} P_{3}^{c_{33}} .
\end{aligned}
$$

Let $\mathbf{W}(\mathbf{P}):=\left(W_{1}(\mathbf{P}), W_{2}(\mathbf{P}), W_{3}(\mathbf{P})\right)$. Let $\mathbf{H}(\mathbf{P})$ be a $3 \times 3$ matrix-valued function of $\mathbf{P} \in \mathbb{R}_{++}^{3}$ defined as

$$
\mathbf{H}(\mathbf{P}):=\left[\begin{array}{ccc}
\frac{1}{P_{1}} & 0 & 0 \\
0 & \frac{1}{P_{2}} & 0 \\
0 & 0 & \frac{1}{P_{3}}
\end{array}\right] \mathbf{C}\left[\begin{array}{ccc}
W_{1}(\mathbf{P}) & 0 & 0 \\
0 & W_{2}(\mathbf{P}) & 0 \\
0 & 0 & W_{3}(\mathbf{P})
\end{array}\right] .
$$

By construction we have

$$
\mathbf{H}(\mathbf{P})=\omega\left[\begin{array}{lll}
c_{11} P_{1}^{c_{11}-1} P_{2}^{c_{21}} P_{3}^{c_{31}} & c_{12} P_{1}^{c_{12}-1} P_{2}^{c_{22}} P_{3}^{c_{32}} & c_{13} P_{1}^{c_{13}-1} P_{2}^{c_{23}} P_{3}^{c_{33}} \\
c_{21} P_{11}^{c_{11}} P_{2}^{c_{1}-1} P_{3}^{c_{31}} & c_{22} P_{1}^{c_{12}} P_{2}^{c_{2}-1} P_{3}^{c_{2}} & c_{23} P_{13}^{c_{13}} P_{23}^{c_{23}-1} P_{33}^{c_{3}} \\
c_{31} P_{1}^{c_{11}} P_{2}^{c_{21}} P_{3}^{c_{31}-1} & c_{32} P_{1}^{c_{12}} P_{2}^{c_{22}} P_{3}^{c_{32}-1} & c_{33} P_{1}^{c_{13}} P_{2}^{c_{23}} P_{3}^{c_{33}-1}
\end{array}\right] .
$$

Since $\mathbf{1 C}=\mathbf{1}$, we also have

$$
\mathbf{W}(\mathbf{P})=\mathbf{P H}(\mathbf{P}) .
$$

Let $\mathbf{0}_{3}:=(0,0,0)^{\mathrm{T}}$. Let $N$ be a set in $\mathbb{R}_{++}^{6}$ defined as

$$
N:=\left\{(\mathbf{K}, \mathbf{P}) \in \mathbb{R}_{++}^{6}: \mathbf{H}(\mathbf{P}) \mathbf{K}^{\mathrm{T}}>\mathbf{0}_{3}\right\} .
$$

At the end of the present subsection we shall show that $N$ is a non-empty open subset of $\mathbb{R}_{++}^{6}$. Let $\mathbf{Y}(\mathbf{K}, \mathbf{P})=$ $\left(Y_{1}(\mathbf{K}, \mathbf{P}), Y_{2}(\mathbf{K}, \mathbf{P}), Y_{3}(\mathbf{K}, \mathbf{P})\right) \in \mathbb{R}_{++}^{3}$ be a $1 \times 3$ vector-valued functions of $(\mathbf{K}, \mathbf{P}) \in N$ defined as

$$
\mathbf{Y}(\mathbf{K}, \mathbf{P})^{\mathrm{T}}:=\mathbf{H}(\mathbf{P}) \mathbf{K}^{\mathrm{T}} .
$$

Let $C\left(P_{1}\right)$ be defined as $C\left(P_{1}\right):=P_{1}^{-\frac{1}{\sigma}}$ for $P_{1}>0$. For $(\mathbf{K}, \mathbf{P}) \in N$, let $K_{i j}(\mathbf{K}, \mathbf{P}), i, j=1,2,3$, be defined as

$$
K_{i j}(\mathbf{K}, \mathbf{P}):=\beta_{i j} \frac{P_{j}}{W_{i}(\mathbf{P})} Y_{j}(\mathbf{K}, \mathbf{P}) .
$$

Then we have

$$
\begin{gathered}
\frac{\partial}{\partial C} \mathscr{H}\left(\mathbf{K}, \mathbf{P}, C\left(P_{1}\right),\left\{K_{i j}\right\}_{i, j=1,2,3}, \mathbf{W}\right)=0 \\
\frac{\partial}{\partial K_{i j}} \mathscr{H}\left(\mathbf{K}, \mathbf{P}, C,\left\{K_{i j}(\mathbf{K}, \mathbf{P})\right\}_{i, j=1,2,3}, \mathbf{W}(\mathbf{P})\right)=0 \\
\sum_{j=1}^{3} K_{i j}(\mathbf{K}, \mathbf{P})=K_{i} .
\end{gathered}
$$

Let $\mathscr{H}^{*}: N \rightarrow \mathbb{R}$ be defined as

$$
\mathscr{H}^{*}(\mathbf{K}, \mathbf{P}):=\mathscr{H}\left(\mathbf{K}, \mathbf{P}, P_{1}^{-\frac{1}{\sigma}},\left\{K_{i j}(\mathbf{K}, \mathbf{P})\right\}_{i, j=1,2,3}, \mathbf{W}(\mathbf{P})\right)
$$


Then by the equalities (4), (5), and (6), $\mathcal{H}^{*}$ is the maximized Hamiltonian of $\mathcal{H}$ for $(\mathbf{K}, \mathbf{P}) \in N$. Since $\mathcal{H}$ is concave in $\left(\mathbf{K}, C,\left\{K_{i j}\right\}_{i, j=1,2,3}\right), \mathcal{H}^{*}$ is concave in $\mathbf{K}$ by the lemma in Kamien and Schwartz (1991, p. 222). Hence we can apply the maximum principle to $\mathscr{H}^{*}=\mathscr{H}^{*}(\mathbf{K}, \mathbf{P})$ for $(\mathbf{K}, \mathbf{P}) \in N$. We obtain the following system of ordinary differential equations and of boundary conditions.

$$
\begin{gathered}
{\left[\begin{array}{c}
\dot{K}_{1} \\
\dot{K}_{2} \\
\dot{K}_{3}
\end{array}\right]=\left(\mathbf{H}\left(P_{1}, P_{2}, P_{3}\right)-g \mathbf{I}_{3}\right)\left[\begin{array}{c}
K_{1} \\
K_{2} \\
K_{3}
\end{array}\right]-\left[\begin{array}{c}
P_{1}^{-\frac{1}{\sigma}} \\
0 \\
0
\end{array}\right]} \\
\dot{P}_{1}=(\rho+g) P_{1}-\omega P_{1}^{c_{11}} P_{2}^{c_{21}} P_{3}^{c_{31}} \\
\dot{P}_{2}=(\rho+g) P_{2}-\omega P_{1}^{c_{12}} P_{2}^{c_{22}} P_{3}^{c_{32}} \\
\dot{P}_{3}=(\rho+g) P_{3}-\omega P_{1}^{c_{13}} P_{2}^{c_{23}} P_{3}^{c_{33}} \\
\left(K_{1}(0), K_{2}(0), K_{3}(0)\right)=\left(\bar{K}_{1}, \bar{K}_{2}, \bar{K}_{3}\right) \\
\lim _{t \rightarrow \infty} e^{-\rho t}\left(P_{1}(t) K_{1}(t)+P_{2}(t) K_{2}(t)+P_{3}(t) K_{3}(t)\right)=0 \\
\forall t \geq 0:(\mathbf{K}(t), \mathbf{P}(t)) \in N,
\end{gathered}
$$

where $K_{i}$ is a predetermined variable, $P_{i}$ is a non-predetermined variable, the condition (9) is an initial condition, and the condition (10) is the transversality condition. The interiority condition (11) guarantees that the maximized Hamiltonian is well defined. If a solution of this system satisfies the summability condition (2), the solution is an optimal solution of the problem (1).

Suppose that an interior BGP exists, and let $\mu_{K}$ and $\mu_{P}$ be balanced growth rates of capital and of its imputed price, respectively. Then we have $\mu_{P}=-\sigma \mu_{K}$ from the equation (7), and $\mu_{P}=-\sigma \mu$ from the equation (8). Thus by Assumption 1.1 $\mu_{K}=\mu>0$, and by Assumption 1.1.b the transversality and summability conditions are satisfied on the BGP.

Before leaving the present subsection, we construct a candidate of an interior BGP of the optimal endogenous growth model (1). Let $\bar{X} \in \mathbb{R}^{3}$ be defined as

$$
\overline{\mathbf{X}}^{\mathrm{T}}:=\left(\omega \mathbf{C}-(g+\mu) \mathbf{I}_{3}\right)^{-1} \mathbf{e}_{1} .
$$

By Lemma $1 \overline{\mathbf{X}}^{\mathrm{T}}>\mathbf{0}_{3}$. Let $\bar{\Lambda}$ be a one-dimensional manifold in $\mathbb{R}_{++}^{6}$ defined as

$$
\bar{\Lambda}:=\left\{(\mathbf{K}, \mathbf{P}) \in \mathbb{R}_{++}^{6}: \exists \lambda>0:(\mathbf{K}, \mathbf{P})=\left(\lambda \overline{\mathbf{X}}, \lambda^{-\sigma} \mathbf{1}\right)\right\} .
$$

Then $\bar{\Lambda} \subset N$, because for $\lambda>0$,

$$
\mathbf{H}\left(\lambda^{-\sigma} \mathbf{1}\right) \lambda \overline{\mathbf{X}}^{\mathrm{T}}=\omega \mathbf{C} \lambda \overline{\mathbf{X}}^{\mathrm{T}}=\lambda\left((g+\mu) \overline{\mathbf{X}}^{\mathrm{T}}+\mathbf{e}_{1}\right)>\mathbf{0}_{3} .
$$

Since $\mathbf{H}(\mathbf{P}) \mathbf{K}^{\mathrm{T}}$ is continuous in $(\mathbf{K}, \mathbf{P}) \in \mathbb{R}_{++}^{6}, N$ is a non-empty open subset of $\mathbb{R}_{++}^{6}$. Therefore $N$ includes some open-neighborhood of $\bar{\Lambda}$. In the next subsection we shall show that $\bar{\Lambda}$ constitutes an interior BGP of the growth model (1).

\subsection{Decomposition}

The present section decomposes the six-dimensional system of equations (7) and (8) into five-dimensional stationary autonomous component and one-dimensional endogenously growing component. Let $f^{1}=f^{1}(x, y)$ and $f^{2}=f^{2}(x, y)$ be functions of $(x, y) \in \mathbb{R}^{2}$ defined as

$$
\begin{aligned}
& f^{1}(x, y):=\omega\left(e^{c_{13} x+c_{23} y}-e^{-\left(c_{21}+c_{31}\right) x+c_{21} y}\right) \\
& f^{2}(x, y):=\omega\left(e^{c_{13} x+c_{23} y}-e^{c_{12} x-\left(c_{12}+c_{32}\right) y}\right) .
\end{aligned}
$$


Let $l=l(x)$ be a function of $x \in \mathbb{R}$ given by the definition (33) in Appendix 2. Let $\mathbf{L}=\mathbf{L}(x, y)$ be a $3 \times 3$ matrix-valued function of $(x, y) \in \mathbb{R}^{2}$ given by the definition (34) in Appendix 2. Let $h=h(x, y)$ be a function of $(x, y) \in \mathbb{R}^{2}$ given by the definition (35) in Appendix 2. By construction each of these functions is sufficiently smooth, $l(0)=0, \mathbf{L}(0,0)=\mathbf{O}_{3}$, and $h(0,0)=0$, where $\mathbf{O}_{3}$ denotes the $3 \times 3$ zero-matrix. Let $\mathbf{e}_{3}:=(0,0,1)^{\mathrm{T}}$. Let $X_{i}, i=1,2, . ., 5$ be defined as

$$
X_{1}:=\frac{P_{1}}{P_{3}}, X_{2}:=\frac{P_{2}}{P_{3}}, X_{3}:=K_{1} P_{3}^{\frac{1}{\sigma}}, X_{4}:=K_{2} P_{3}^{\frac{1}{\sigma}}, X_{5}:=K_{3} P_{3}^{\frac{1}{\sigma}} \text {. }
$$

Let $k_{i}:=\log K_{i}$, and $p_{i}:=\log P_{i}, i=1,2,3$. Let $x_{i}:=\log X_{i}, i=1,2, . ., 5$. Then by the relation (36) in Appendix 2 the six-dimensional system of equations (7) and (8) are decomposed into the following three components.

$$
\begin{gathered}
{\left[\begin{array}{c}
\dot{x}_{1} \\
\dot{x}_{1}
\end{array}\right]=\left[\begin{array}{l}
f^{1}\left(x_{1}, x_{2}\right) \\
f^{2}\left(x_{1}, x_{2}\right)
\end{array}\right]} \\
{\left[\begin{array}{c}
\dot{X}_{3} \\
\dot{X}_{4} \\
\dot{X}_{5}
\end{array}\right]=\left(\omega \mathbf{C}-(g+\mu) \mathbf{I}_{3}\right)\left[\begin{array}{c}
X_{3} \\
X_{4} \\
X_{5}
\end{array}\right]-\mathbf{e}_{1}+\mathbf{L}\left(x_{1}, x_{2}\right)\left[\begin{array}{c}
X_{3} \\
X_{4} \\
X_{5}
\end{array}\right]} \\
-l\left(-\frac{1}{\sigma} x_{1}\right) \mathbf{e}_{1}-\frac{\omega}{\sigma} h\left(x_{1}, x_{2}\right)\left[\begin{array}{c}
X_{3} \\
X_{4} \\
X_{5}
\end{array}\right] \\
\dot{k}_{3}=\mathbf{e}_{3}^{\mathrm{T}} \omega \mathbf{C}\left[\begin{array}{c}
X_{3} \\
X_{5} \\
\frac{X_{4}}{X_{5}} \\
1
\end{array}\right]-g+\mathbf{e}_{3}^{\mathrm{T}} \mathbf{L}\left(x_{1}, x_{2}\right)\left[\begin{array}{c}
\frac{X_{3}}{X_{5}} \\
\frac{X_{4}}{X_{5}} \\
1
\end{array}\right] .
\end{gathered}
$$

The system (14) is a two-dimensional stationary autonomous component. The system composed of the differential equations (14) and (15) is a five-dimensional stationary autonomous component. The equation (16) is one-dimensional endogenously growing component.

Let $\left(x_{1}^{*}, x_{2}^{*}, X_{3}^{*}, X_{4}^{*}, X_{5}^{*}\right)$ be defined as

$$
\left(x_{1}^{*}, x_{2}^{*}, X_{3}^{*}, X_{4}^{*}, X_{5}^{*}\right):=(0,0, \overline{\mathbf{X}}),
$$

where $\overline{\mathbf{X}}$ is given by the definition (12). Then $\left(x_{1}^{*}, x_{2}^{*}, X_{3}^{*}, X_{4}^{*} X_{5}^{*}\right)$ is a steady state of the five-dimensional autonomous stationary system composed of the differential equations (14) and (15). Since $\overline{\mathbf{X}}^{\mathrm{T}}>\mathbf{0}_{3}$ by Lemma 1 , $x_{i}^{*}=\log X_{i}^{*}, i=3,4,5$ are well defined. Let $\mathbf{T}(\sigma)$ be the $5 \times 6$ matrix given by the definition (37) in Appendix 3 . The rank of $\mathbf{T}(\sigma)$ is five. Consider the following one-dimensional manifold $\Lambda^{*}$ in $\mathbb{R}_{++}^{6}$.

$$
\Lambda^{*}:=\left\{(\mathbf{K}, \mathbf{P}) \in \mathbb{R}_{++}^{6}: \mathbf{T}(\sigma)\left[\begin{array}{c}
k_{1} \\
k_{2} \\
k_{3} \\
p_{1} \\
p_{2} \\
p_{3}
\end{array}\right]=\left[\begin{array}{c}
0 \\
0 \\
x_{3}^{*} \\
x_{4}^{*} \\
x_{5}^{*}
\end{array}\right]\right\}
$$

By the relation (38) in Appendix 3, the manifold $\Lambda^{*}$ constitutes a BGP of the optimal growth model (1). We obtain from the construction of $\mathbf{T}(\sigma),(\mathbf{K}, \mathbf{P}) \in \Lambda^{*}$, if and only if $(\mathbf{K}, \mathbf{P})=\left(\lambda \overline{\mathbf{X}}, \lambda^{-\sigma} \mathbf{1}\right)$ for $\lambda=P_{3}^{-\frac{1}{\sigma}}$. Therefore we have

$$
\Lambda^{*}=\bar{\Lambda} \subset N,
$$

where $\bar{\Lambda}$ and $N$ are the sets given by the definitions (13) and (3). Since $\Lambda^{*} \subset N$, and since $\mathbf{H}(\mathbf{P}) \mathbf{K}^{\mathrm{T}}>\mathbf{0}_{3}$ for $(\mathbf{K}, \mathbf{P}) \in N$, the interiority condition (11) is satisfied on $\Lambda^{*}$ and on some open neighborhood of it. As mentioned in the previous subsection, by Assumption 1.1.b the transversality and the summability conditions are satisfied on $\Lambda^{*}$. Thus we have the following proposition. 


\section{Proposition 1}

Suppose that Assumption 1 is satisfied. The optimal growth model (1) has an interior equilibrium BGP.

Before leaving the present subsection, consider the following set $N_{1}$ in $\mathbb{R}^{2} \times \mathbb{R}_{++}^{3}$ for the later use.

$$
N_{1}:=\left\{\left(\mathbf{x}_{1}, \mathbf{X}_{2}\right) \in \mathbb{R}^{2} \times \mathbb{R}_{++}^{3}:\left(\omega C+L\left(\mathbf{x}_{1}\right)\right) \mathbf{X}_{2}^{\mathrm{T}}>\mathbf{0}_{3}\right\} .
$$

By construction, $\mathbf{H}(\mathbf{P}) \mathbf{K}^{\mathrm{T}}>\mathbf{0}_{3}$ if and only if $\left(\omega C+L\left(\mathbf{x}_{1}\right)\right) \mathbf{X}_{2}^{\mathrm{T}}>\mathbf{0}_{3} \wedge P_{3}>0$ with $\left(\mathbf{x}_{1}, \mathbf{X}_{2}\right)=\left(x_{1}, x_{2}, X_{3}, X_{4}, X_{5}\right)$. Therefore, $(\mathbf{K}, \mathbf{P}) \in N$, if and only if $\left(x_{1}, x_{2}, X_{3}, X_{4}, X_{5}\right) \in N_{1} \wedge P_{3}>0$. The set $N_{1}$ includes the steady state $\left(0,0, X_{3}^{*}, X_{4}^{*}, X_{5}^{*}\right)$ and some open neighborhood of it. Consider also the following set $N_{2}$ in $\mathbb{R}^{5}$ for the later use.

$$
N_{2}:=\left\{\left(x_{1}, x_{2}, x_{3}, x_{4}, x_{5}\right) \in \mathbb{R}^{5}:\left(x_{1}, x_{2}, e^{x_{3}}, e^{x_{4}}, e^{x_{5}}\right) \in N_{1}\right\} .
$$

Then by construction, $(\mathbf{K}, \mathbf{P}) \in N$, if and only if $\left(x_{1}, x_{2}, x_{3}, x_{4}, x_{5}\right) \in N_{2} \wedge P_{3}>0$. The set $N_{2}$ includes the point $\left(0,0, x_{3}^{*}, x_{4}^{*}, x_{5}^{*}\right)$ and some open neighborhood of it.

\subsection{Transitional dynamics}

The present section considers the transitional dynamics and the local determinacy of equilibrium around the BGP $\Lambda^{*}$ in terms of predetermined and non-predetermined variables. Let $z_{i}, i=1,2$, and $q_{i}, i=1,2,3$ be variables given by the definition (39) in Appendix 3. And let $\mathbf{M}(\sigma)$ be the $5 \times 5$ matrix given by the definition (40) in Appendix 3. Then $\operatorname{det} \mathbf{M}(\sigma) \neq 0$, and we have $\left(z_{1}, z_{2}, q_{1}, q_{2}, q_{3}\right)=\left(x_{1}, x_{2}, x_{3}, x_{4}, x_{5}\right) \mathbf{M}(\sigma)^{\mathrm{T}}$ and $\left(x_{1}, x_{2}, x_{3}, x_{4}, x_{5}\right)=$ $\left(z_{1}, z_{2}, q_{1}, q_{2}, q_{3}\right)\left(\mathbf{M}(\sigma)^{-1}\right)^{\mathrm{T}}$. See the relation (41) in Appendix 3. By construction $z_{i}, i=1,2$ are predetermined variables and $q_{i}, i=1,2,3$ are non-predetermined variables. Let $\left(z_{1}^{*}, z_{2}^{*}, q_{1}^{*}, q_{2}^{*}, q_{3}^{*}\right)$ be defined as

$$
\left(z_{1}^{*}, z_{2}^{*}, q_{1}^{*}, q_{2}^{*}, q_{3}^{*}\right):=\left(0,0, x_{3}^{*}, x_{4}^{*}, x_{5}^{*}\right) \mathbf{M}(\sigma)^{\mathrm{T}} .
$$

Then $\left(z_{1}^{*}, z_{2}^{*}, q_{1}^{*}, q_{2}^{*}, q_{3}^{*}\right)\left(\mathbf{M}(\sigma)^{-1}\right)^{\mathrm{T}} \in N_{2}$, and let $N_{3}$ be a set in $\mathbb{R}^{5}$ defined as

$$
N_{3}:=\left\{\left(z_{1}, z_{2}, q_{1}, q_{2}, q_{3}\right) \in \mathbb{R}^{5}:\left(z_{1}, z_{2}, q_{1}, q_{2}, q_{3}\right)\left(\mathbf{M}(\sigma)^{-1}\right)^{\mathrm{T}} \in N_{2}\right\} .
$$

Then $\left(z_{1}^{*}, z_{2}^{*}, q_{1}^{*}, q_{2}^{*}, q_{3}^{*}\right) \in N_{3}$, and the set $N_{3}$ includes some open neighborhood of it. By construction, $(\mathbf{K}, \mathbf{P}) \in N$, if and only if $\left(z_{1}, z_{2}, q_{1}, q_{2}, q_{3}\right) \in N_{3} \wedge P_{3}>0$. Therefore in some open neighborhood of $\left(z_{1}^{*}, z_{2}^{*}, q_{1}^{*}, q_{2}^{*}, q_{3}^{*}\right)$, the maximized Hamiltonian is well defined, and local dynamics near the steady state $\left(z_{1}^{*}, z_{2}^{*}, q_{1}^{*}, q_{2}^{*}, q_{3}^{*}\right)$ is also well defined.

Let $\mathbf{J}$ be a $2 \times 2$ matrix defined as

$$
\mathbf{J}:=\omega\left[\begin{array}{cc}
c_{13}+c_{21}+c_{31} & -\left(c_{21}-c_{23}\right) \\
c_{13}-c_{12} & c_{23}+c_{12}+c_{32}
\end{array}\right] .
$$

Then the characteristic roots of the five-dimensional autonomous stationary system composed of the differential equations (14) and (15) evaluated at the steady state $(0,0, \overline{\mathbf{X}})$ are given by two characteristic roots of $\mathbf{J}$ and three characteristic roots of $\omega \mathbf{C}-(g+\mu) \mathbf{I}_{3}$. Consider Example 1, Example 2, Example 3 in Section 2. Each of these examples has two stable roots and three unstable roots. $\left(x_{1}, x_{2}, x_{3}, x_{4}, x_{5}\right)$ one to one corresponds to $\left(z_{1}, z_{2}, q_{1}, q_{2}, q_{3}\right)$ that includes two predetermined and three non-predetermined variables. Thus in each of these examples, the interior BGP is saddle point stable.

\section{Observation 1}

For some parameter values of $(\rho, \sigma, \mu, g, \mathbf{B})$, the optimal growth model (1) has an interior equilibrium BGP that is saddle point stable.

Since $\left(z_{1}, z_{2}, q_{1}, q_{2}, q_{3}\right)$ includes two predetermined variables $z_{i}, i=1,2$, the stationary autonomous dynamics (14) and (15) might have a closed orbit that is locally determinate and locally stable around an unstable BGP. In other words, the BGP might not always be saddle point stable. For $\eta>0$, let $\mathbf{B}(\eta)$ be a $3 \times 3$ matrix defined as 


$$
\overline{\mathbf{B}}(\eta):=\left[\begin{array}{ccc}
1-\frac{(2+3 \eta)(2+2 \eta)}{5+12 \eta+9 \eta^{2}} & \frac{(2+3 \eta)(1+\eta)}{5+12 \eta+9 \eta^{2}} & \frac{(2+3 \eta) \eta}{5+12 \eta+9 \eta^{2}} \\
\frac{(2+3 \eta) \eta}{5+12 \eta+9 \eta^{2}} & 1-\frac{(2+3 \eta)(1+2 \eta)}{5+12 \eta+9 \eta^{2}} & \frac{(2+3 \eta)(1+\eta)}{5+12 \eta+9 \eta^{2}} \\
\frac{(2+3 \eta)(2+\eta)}{5+12 \eta+9 \eta^{2}} & \frac{(2+3 \eta) \eta}{5+12 \eta+9 \eta^{2}} & 1-\frac{(2+3 \eta)(1+2 \eta)}{5+12 \eta+9 \eta^{2}}
\end{array}\right]
$$

Each element of $\overline{\mathbf{B}}(\eta)$ is positive and less than 1 , and $\overline{\mathbf{1}} \overline{\mathbf{B}}(\eta)=\mathbf{1}$. We have

$$
\operatorname{det} \overline{\mathbf{B}}(\eta)=\frac{1}{5+12 \eta+9 \eta^{2}}>0 .
$$

Let $\bar{c}_{i j}=\bar{c}_{i j}(\eta)$ be the $(i, j)$-element of the inverse matrix of $\overline{\mathbf{B}}(\eta)$ for each $i, j=1,2,3$. Then we have

$$
\omega\left[\begin{array}{cc}
\bar{c}_{13}+\bar{c}_{21}+\bar{c}_{31} & -\left(\bar{c}_{21}-\bar{c}_{23}\right) \\
\bar{c}_{13}-\bar{c}_{12} & \bar{c}_{23}+\bar{c}_{12}+\bar{c}_{32}
\end{array}\right]=\omega\left[\begin{array}{cc}
0 & -(2+3 \eta) \\
2+3 \eta & 0
\end{array}\right] .
$$

And the corresponding autonomous dynamics (14) has a pair of pure imaginary complex conjugate roots at a steady state. ${ }^{5}$ This suggests that the system (14) undergoes Hopf bifurcation under perturbations of the technology matrix $\overline{\mathbf{B}}(\eta)$. We conduct an exact bifurcation analysis in the next section.

\section{Existence and stability of closed orbit}

\subsection{Hopf bifurcation}

The present subsection applies the Hopf bifurcation theorem to the two-dimensional autonomous system (14). For $\eta>0$, let $\overline{\mathbf{B}}(\eta)$ be the $3 \times 3$ matrix given by the definition (17). For $\eta>0$ and for $v$ in some neighborhood of 0 , let $\mathbf{B}(\nu, \eta)$ be a $3 \times 3$ matrix defined as

$$
\mathbf{B}(\nu, \eta):=\overline{\mathbf{B}}(\eta)+\left[\begin{array}{ccc}
-v & 0 & 0 \\
v & 0 & 0 \\
0 & 0 & 0
\end{array}\right] .
$$

As $\nu$ varies around 0 , the matrix $\mathbf{B}(\nu, \eta)$ generates perturbations of the matrix $\overline{\mathbf{B}}(\eta)$. We have $\mathbf{1 B}(\nu, \eta)=\mathbf{1}$ and

$$
\operatorname{det} \mathbf{B}(\nu, \eta)=\frac{1-3(1+\eta) v}{5+12 \eta+9 \eta^{2}}
$$

Suppose $\eta>0$ and $1-3(1+\eta) v \neq 0$. Let $\mathbf{C}(\nu, \eta)$ denote the inverse matrix of $\mathbf{B}(v, \eta)$.

$$
\mathbf{C}(\nu, \eta):=\mathbf{B}(\nu, \eta)^{-1} \text {. }
$$

Let $c_{i j}(\nu, \eta)$ denote the $(i, j)$-element of $\mathbf{C}(\nu, \eta)$ for each $i, j=1,2,3$. Let $\mathbf{J}(\nu, \eta, \omega)$ be a $2 \times 2$ matrix defined as

$$
\mathbf{J}(\nu, \eta, \omega):=\omega\left[\begin{array}{cc}
c_{13}(\nu, \eta)+c_{21}(\nu, \eta)+c_{31}(\nu, \eta) & -\left(c_{21}(\nu, \eta)-c_{23}(\nu, \eta)\right) \\
c_{13}(\nu, \eta)-c_{12}(\nu, \eta) & c_{23}(\nu, \eta)+c_{12}(\nu, \eta)+c_{32}(\nu, \eta)
\end{array}\right] .
$$

Then we obtain the following relation.

$$
\mathbf{J}(\nu, \eta, \omega)=\omega\left[\begin{array}{cc}
-\frac{3(1+\eta)}{1-3(1+\eta) \nu} \nu & -\frac{2+3 \eta}{1-3(1+\eta) v}+\frac{5+12 \eta+9 \eta^{2}}{1-3(1+\eta) \nu} v \\
\frac{2+3 \eta}{1-3(1+\eta) \nu} & \frac{2+9 \eta+9 \eta^{2}}{1-3(1+\eta) \nu} v
\end{array}\right] .
$$

And we have

$$
\left.\frac{d}{d v}[\operatorname{tr} \mathbf{J}(\nu, \eta, \omega)]\right|_{\nu=0}=\omega\left(9 \eta^{2}+6 \eta-1\right)
$$

By construction the following four relations hold for $\eta>0$. 
1. If $-\frac{2 \eta+3 \eta^{2}}{5+12 \eta+9 \eta^{2}}<v<\frac{1+2 \eta+3 \eta^{2}}{5+12 \eta+9 \eta^{2}}$, then $0<1-\frac{(2+3 \eta)(2+2 \eta)}{5+12 \eta+9 \eta^{2}}-v<1$, and $0<\frac{(2+3 \eta) \eta}{5+12 \eta+9 \eta^{2}}+v<1$.

2. If $v<\frac{1}{3(1+\eta)}$, then $\operatorname{det} \mathbf{B}(\nu, \eta)>0$.

3. If $-\frac{2(1+\sqrt{2})(2+3 \eta)}{5+12 \eta+9 \eta^{2}}<v<\frac{2(\sqrt{2}-1)(2+3 \eta)}{5+12 \eta+9 \eta^{2}}$, then the characteristic roots of $\mathbf{J}(\nu, \eta, \omega)$ are a pair of conjugate complex roots.

4. If $\eta \neq \frac{\sqrt{2}-1}{3}$, then $\left.\frac{d}{d \nu}[\operatorname{tr} \mathbf{J}(\nu, \eta, \omega)]\right|_{\nu=0} \neq 0$.

Let $v_{1}=v_{1}(\eta)$ and $v_{2}=v_{2}(\eta)$ be functions of $\eta>0$ defined as

$$
\begin{aligned}
& \nu_{1}(\eta):=\frac{2+3 \eta}{5+12 \eta+9 \eta^{2}} \min \{\eta, 2(1+\sqrt{2})\} \\
& \nu_{2}(\eta):=\min \left\{\frac{1+2 \eta+3 \eta^{2}}{5+12 \eta+9 \eta^{2}}, \frac{1}{3(1+\eta)}, \frac{2(\sqrt{2}-1)(2+3 \eta)}{5+12 \eta+9 \eta^{2}}\right\},
\end{aligned}
$$

where $\min \{x, y\}$ and $\min \{x, y, z\}$ denote the minimum element of each set. In the rest of the paper we make the following assumption.

\section{Assumption 2}

1. $\eta>0$ and $\eta \neq \frac{\sqrt{2}-1}{3}$.

2. $-v_{1}(\eta)<v<v_{2}(\eta)$.

Let $f^{1}(\eta, \omega)=f^{1}(x, y, v, \eta, \omega)$ and $f^{2}(\eta, \omega)=f^{2}(x, y, v, \eta, \omega)$ be functions of $(x, y, v) \in \mathbb{R}^{2} \times\left(-v_{1}(\eta), \nu_{2}(\eta)\right)$ defined as

$$
\begin{aligned}
& f^{1}(x, y, \nu, \eta, \omega):=\omega\left(e^{c_{13}(\nu, \eta) x+c_{23}(\nu, \eta) y}-e^{-\left(c_{21}(\nu, \eta)+c_{31}(\nu, \eta)\right) x+c_{21}(v, \eta) y}\right) \\
& f^{2}(x, y, v, \eta, \omega):=\omega\left(e^{c_{13}(\nu, \eta) x+c_{23}(v, \eta) y}-e^{c_{12}(\nu, \eta) x-\left(c_{12}(\nu, \eta)+c_{32}(v, \eta)\right) y}\right) .
\end{aligned}
$$

Consider the following one-parameter family of ordinary differential equations parametrized by $v \in$ $\left(-v_{1}(\eta), v_{2}(\eta)\right)$.

$$
\left[\begin{array}{c}
\dot{x}_{1} \\
\dot{x}_{2}
\end{array}\right]=\left[\begin{array}{l}
f^{1}\left(x_{1}, x_{2}, v, \eta, \omega\right) \\
f^{2}\left(x_{1}, x_{2}, v, \eta, \omega\right)
\end{array}\right] .
$$

Each element of this family is the two-dimensional autonomous system (14) with $\mathbf{C}=\mathbf{B}(\nu, \eta)^{-1}$. The Jacobian matrix of the right hand side of the differential equation (22) evaluated at a steady state $\left(x_{1}, x_{2}\right)=(0,0)$ is given by $\mathbf{J}(\nu, \eta, \omega)$. And we have

$$
\mathbf{J}(0, \eta, \omega)=\left[\begin{array}{cc}
0 & -(2+3 \eta) \omega \\
(2+3 \eta) \omega & 0
\end{array}\right] .
$$

By Assumption $2 \mathbf{J}(\nu, \eta, \omega)$ has a pair of conjugate complex roots as its characteristic roots. Let $\lambda(\nu, \eta, \omega)$ and $\bar{\lambda}(\nu, \eta, \omega)$ be conjugate complex characteristic rotos of $\mathbf{J}(\nu, \eta, \omega)$, and let $\operatorname{Re}(\lambda(\nu, \eta, \omega))$ be the real part of $\lambda(\nu, \eta, \omega)$. Then we obtain

$$
\left.\frac{d}{d \nu} \operatorname{Re}(\lambda(\nu, \eta, \omega))\right|_{\nu=0}=\frac{\omega}{2}\left(9 \eta^{2}+6 \eta-1\right)
$$

By Assumption 2.1 we have

$$
\left.\frac{d}{d \nu} \operatorname{Re}(\lambda(\nu, \eta, \omega))\right|_{\nu=0} \neq 0
$$

Let $a=a(\eta, \omega)$ be a number defined by the formula (42) in Appendix 4. Let $\hat{a}=\hat{a}(\eta, \omega)$ be a number defined by the formula (43) in Appendix 5. Then one can derive the following relation from routine, albeit tedious, calculations under Assumption 2.1. 


$$
a(\eta, \omega)=\hat{a}(\eta, \omega) .
$$

Therefore the following proposition holds by the Hopf bifurcation theorem (Guckenheimer and Holmes [1983, Theorem 3.4.2]).

\section{Proposition 2}

Suppose that Assumption 2 is satisfied. Let $a=a(\eta, \omega)$ be a number defined by the formula (42). If $a<0$, the system (22) undergoes supercritical Hopf bifurcation at $v=0$. If $a>0$, the system (22) undergoes subcritical Hopf bifurcation at $\nu=0$.

The following three examples satisfy Assumption 2. Recall $\omega:=\rho+g+\sigma \mu$. In each example, $\left.\frac{d}{d v} \operatorname{Re}(\lambda(\nu, \eta))\right|_{\nu=0}>0$, and $a(\eta, \omega)<0$. Thus as $\nu$ increases and crosses 0 , a locally unique steady state $\left(x_{1}, x_{2}\right)=(0,0)$ loses its stability, and the system (22) undergoes supercritical Hopf bifurcation.

\section{Example 4}

Let $\rho=\frac{5}{100}, \sigma=\frac{5}{100}, \mu=\frac{3}{100}, g=\frac{2}{100}$, and $\eta=1$. Then one obtains

$$
\begin{gathered}
\overline{\mathbf{B}}(\eta)=\left[\begin{array}{ccc}
\frac{3}{13} & \frac{5}{13} & \frac{5}{26} \\
\frac{5}{26} & \frac{11}{26} & \frac{5}{13} \\
\frac{15}{26} & \frac{5}{26} & \frac{11}{26}
\end{array}\right] \\
\mathbf{J}(0, \eta, \omega)=\left[\begin{array}{cc}
0 & -\frac{143}{400} \\
\frac{143}{400} & 0
\end{array}\right] \\
\frac{d}{d \nu} \operatorname{Re}\left(\left.\lambda(\nu, \eta, \omega)\right|_{\nu=0}=\frac{1001}{2000}, \text { and } a(\eta, \omega)=-\frac{11}{256} .\right.
\end{gathered}
$$

\section{Example 5}

Let $\rho=\frac{5}{100}, \sigma=\frac{1}{10}, \mu=\frac{2}{100}, g=\frac{2}{100}$, and $\eta=\frac{1}{2}$. Then one obtains

$$
\begin{gathered}
\overline{\mathbf{B}}(\eta)=\left[\begin{array}{ccc}
\frac{11}{53} & \frac{21}{53} & \frac{7}{53} \\
\frac{7}{53} & \frac{25}{53} & \frac{21}{53} \\
\frac{35}{53} & \frac{7}{53} & \frac{25}{53}
\end{array}\right] \\
\mathbf{J}(0, \eta, \omega)=\left[\begin{array}{cc}
0 & -\frac{63}{250} \\
\frac{63}{250} & 0
\end{array}\right] \\
\left.\frac{d}{d v} \operatorname{Re}(\lambda(\nu, \eta, \omega))\right|_{\nu=0}=\frac{153}{1000}, \text { and } a(\eta, \omega)=-\frac{9261}{424000} .
\end{gathered}
$$

Example 6

Let $\rho=\frac{5}{100}, \sigma=\frac{3}{2}, \mu=\frac{3}{100}, g=\frac{2}{100}$, and $\eta=3$. Then one obtains

$$
\overline{\mathbf{B}}(\eta)=\left[\begin{array}{ccc}
\frac{17}{61} & \frac{22}{61} & \frac{33}{122} \\
\frac{33}{122} & \frac{45}{122} & \frac{22}{61} \\
\frac{55}{122} & \frac{33}{122} & \frac{45}{122}
\end{array}\right]
$$




$$
\begin{aligned}
\mathbf{J}(0, \eta, \omega) & =\left[\begin{array}{cc}
0 & -\frac{253}{200} \\
\frac{253}{200} & 0
\end{array}\right] \\
\left.\frac{d}{d \nu} \operatorname{Re}(\lambda(\nu, \eta, \omega))\right|_{\nu=0} & =\frac{1127}{200}, \text { and } a(\eta, \omega)=-\frac{30613}{97600} .
\end{aligned}
$$

The following example satisfies Assumption 2. In this example, $\left.\frac{d}{d v} \operatorname{Re}(\lambda(\nu, \eta))\right|_{\nu=0}<0$, and $a(\eta, \omega)<0$. Thus as $v$ decreases and crosses 0 , a locally unique steady state $\left(x_{1}, x_{2}\right)=(0,0)$ loses its stability, and the system (22) undergoes supercritical Hopf bifurcation.

\section{Example 7}

Let $\rho=\frac{5}{100}, \sigma=\frac{3}{2}, \mu=\frac{3}{100}, g=\frac{2}{100}$, and $\eta=\frac{1}{10}$. Then one obtains

$$
\begin{gathered}
\overline{\mathbf{B}}(\eta)=\left[\begin{array}{ccc}
\frac{123}{629} & \frac{253}{629} & \frac{23}{629} \\
\frac{23}{629} & \frac{353}{629} & \frac{253}{629} \\
\frac{483}{629} & \frac{23}{629} & \frac{353}{629}
\end{array}\right] \\
\mathbf{J}(0, \eta, \omega)=\left[\begin{array}{cc}
0 & -\frac{529}{2000} \\
\frac{529}{2000} & 0
\end{array}\right] \\
\left.\frac{d}{d \nu} \operatorname{Re}(\lambda(\nu, \eta, \omega))\right|_{\nu=0}=-\frac{713}{40000}, \text { and } a(\eta, \omega)=-\frac{3078251}{201280000} .
\end{gathered}
$$

\subsection{Center manifold reduction}

The present subsection constructs one-parameter family of optimal growth models parametrized by $\nu$ based on the optimal growth model (1), and applies the center manifold reduction to equilibrium dynamics of this parametrized family of models and obtains a bifurcation diagram in order to analyze the stability and the determinacy of equilibrium around a closed orbit obtained by supercritical Hopf bifurcation.

Let $\Gamma, \Pi$, and $\Theta$ be defined as

$$
\begin{aligned}
& \Gamma:=\left\{\eta \in \mathbb{R}_{++}: \eta \neq \frac{\sqrt{2}-1}{3}\right\} \\
& \Pi:=\left\{(\rho, \sigma, \mu, g) \in \mathbb{R}_{++}^{3} \times \mathbb{R}_{+}: \rho-(1-\sigma) \mu>0\right\} \\
& \Theta:=\Gamma \times \Pi .
\end{aligned}
$$

For $\eta \in \Gamma$, let $\bar{\beta}_{i j}(\eta)$ denote the $(i, j)$-element of $\overline{\mathbf{B}}(\eta)$. For $\eta \in \Gamma$ and for $v \in\left(-v_{1}(\eta), v_{2}(\eta)\right)$, let $\beta_{i j}(\nu, \eta)$ denote the $(i, j)$-element of $\mathbf{B}(\nu, \eta)$. For $\eta \in \Gamma$ and for $v \in\left(-v_{1}(\eta), v_{2}(\eta)\right)$, let $b_{1}(\nu, \eta), b_{2}(\eta)$, and $b_{3}(\eta)$ be defined as

$$
\begin{aligned}
b_{1}(\nu, \eta) & :=\left(\beta_{11}(\nu, \eta)\right)^{\beta_{11}(v, \eta)}\left(\beta_{21}(\nu, \eta)\right)^{\beta_{21}(v, \eta)}\left(\bar{\beta}_{31}(\eta)\right)^{\bar{\beta}_{31}(\eta)} \\
b_{2}(\eta) & :=\left(\bar{\beta}_{12}(\eta)\right)^{\bar{\beta}_{12}(\eta)}\left(\bar{\beta}_{22}(\eta)\right)^{\bar{\beta}_{22}(\eta)}\left(\bar{\beta}_{32}(\eta)\right)^{\bar{\beta}_{32}(\eta)} \\
b_{3}(\eta) & :=\left(\bar{\beta}_{13}(\eta)\right)^{\bar{\beta}_{13}(\eta)}\left(\bar{\beta}_{23}(\eta)\right)^{\bar{\beta}_{23}(\eta)}\left(\bar{\beta}_{33}(\eta)\right)^{\bar{\beta}_{33}(\eta)} .
\end{aligned}
$$

For $\pi \in \Pi$, let $\omega(\pi)$ be defined as

$$
\omega(\pi):=\rho+g+\sigma \mu
$$

For $\theta=(\eta, \pi) \in \Theta$ and for $v \in\left(-v_{1}(\eta), v_{2}(\eta)\right)$, let $e_{1}(\nu, \theta), e_{2}(\theta)$ and $e_{3}(\theta)$ be defined as 


$$
e_{1}(\nu, \theta):=\frac{\omega(\pi)}{b_{1}(\nu, \eta)}, e_{2}(\theta):=\frac{\omega(\pi)}{b_{2}(\eta)}, e_{3}(\theta)=\frac{\omega(\pi)}{b_{3}(\eta)} .
$$

Then Assumption 1 and Assumption 2 are satisfied for a given $\theta=(\eta, \pi) \in \Theta$ and for each $v \in\left(-v_{1}(\eta), v_{2}(\eta)\right)$. In the rest of the present subsection we suppose that

$$
\theta=(\eta, \pi) \in \Theta=\Gamma \times \Pi .
$$

We have the following lemma. See Appendix 6 for the proof.

\section{Lemma 2}

Suppose that $(\eta, \pi) \in \Theta$. Then each characteristic root of $\omega(\pi) \mathbf{C}(0, \eta)-(g+\mu) \mathbf{I}_{3}$ has a strictly positive real part.

Consider the following one-parameter family of optimal growth models parametrized by $v \in$ $\left(-v_{1}(\eta), v_{2}(\eta)\right)$.

$$
\operatorname{Max}_{C, K_{i j}, i, j=1,2,3} \int_{0}^{\infty} \frac{C^{1-\sigma}-1}{1-\sigma} e^{-\rho t} d t
$$

subject to

$$
\begin{gathered}
C>0, K_{i j} \geq 0, i, j=1,2,3, K_{i}>0, i=1,2,3, \\
\dot{K}_{1}=e_{1}(\nu, \theta)\left(K_{11}\right)^{\beta_{11}(v, \eta)}\left(K_{21}\right)^{\beta_{21}(v, \eta)}\left(K_{31}\right)^{\bar{\beta}_{31}(\eta)}-C-g K_{1} \\
\dot{K}_{2}=e_{2}(\theta)\left(K_{12}\right)^{\bar{\beta}_{12}(\eta)}\left(K_{22}\right)^{\bar{\beta}_{22}(\eta)}\left(K_{32} \bar{\beta}_{32}(\eta)-g K_{2}\right. \\
\dot{K}_{3}=e_{3}(\theta)\left(K_{13}\right)^{\bar{\beta}_{13}(\eta)}\left(K_{23}\right)^{\bar{\beta}_{23}(\eta)}\left(K_{33}\right)^{\bar{\beta}_{33}(\eta)}-g K_{3} \\
K_{11}+K_{12}+K_{13}=K_{1} \\
K_{21}+K_{22}+K_{23}=K_{2} \\
K_{31}+K_{32}+K_{33}=K_{3} \\
K_{1}(0)=\bar{K}_{1}>0, K_{2}(0)=\bar{K}_{2}>0, K_{3}(0)=\bar{K}_{3}>0 .
\end{gathered}
$$

By Lemma 1 for $v \in\left(-v_{1}(\eta), v_{2}(\eta)\right)$, $\operatorname{det}\left(\omega(\pi) \mathbf{C}(\nu, \eta)-(g+\mu) \mathbf{I}_{3}\right) \neq 0$, and each component of $\left(\omega(\pi) \mathbf{C}(\nu, \eta)-(g+\mu) \mathbf{I}_{3}\right)^{-1} \mathbf{e}_{1}$ is strictly positive. Let $\mathbf{X}^{*}(\nu, \theta) \in \mathbb{R}_{++}^{3}$ be defined as

$$
\mathbf{X}^{*}(\nu, \theta)^{\mathrm{T}}:=\left(\omega(\pi) \mathbf{C}(\nu, \eta)-(g+\mu) \mathbf{I}_{3}\right)^{-1} \mathbf{e}_{1} .
$$

Recall that $c_{i j}(\nu, \eta)$ is the $(i, j)$-element of $\mathbf{C}(\nu, \eta)$. Since $\operatorname{det} \mathbf{B}(\nu, \eta)>0, c_{i j}(\nu, \eta)$ is at least twice continuously differentiable relative to $\nu$. Let $f^{i}(\eta, \omega)=f^{i}(x, y, v, \eta, \omega), i=1,2$, be functions of $(x, y, v) \in \mathbb{R}^{2} \times\left(-v_{1}(\eta), v_{2}(\eta)\right)$ given by the defining functions (21). Then each of $f^{i}(x, y, v, \eta, \omega(\pi)), i=1,2$, is at least twice continuously differentiable relative to $(x, y, v)$ with $f^{i}(0,0, v, \eta, \omega(\pi))=0$. Substitute $c_{i j}(v, \eta), i, j=1,2,3$ for each $c_{i j}$ that appears in the defining functions (34) and (35) in Appendix 2, and denote the functions thus obtained by $\mathbf{L}(\nu, \eta, \omega)=\mathbf{L}(x, y, v, \eta, \omega)$ and $h(v, \eta)=h(x, y, v, \eta)$. Then each of $\mathbf{L}(x, y, v, \eta, \omega(\pi))$ and $h(x, y, v, \eta)$ is at least twice continuously differentiable relative to $(x, y, v) \in \mathbb{R}^{2} \times\left(-v_{1}(\eta), v_{2}(\eta)\right)$ with $\mathbf{L}(0,0, v, \eta, \omega(\pi))=\mathbf{O}_{3}$ and with $h(0,0, v, \eta)=0$. Let $l=l(x)$ be an analytical function given by the defining function (33) in Appendix 2 . By construction $l(0)=0$.

Consider the following six-dimensional system of autonomous differential equations.

$$
\begin{aligned}
& {\left[\begin{array}{c}
\dot{x}_{1} \\
\dot{x}_{2}
\end{array}\right]=\left[\begin{array}{l}
f^{1}\left(x_{1}, x_{2}, v, \eta, \omega(\pi)\right) \\
f^{2}\left(x_{1}, x_{2}, v, \eta, \omega(\pi)\right)
\end{array}\right] } \\
& {\left[\begin{array}{c}
\dot{X}_{3} \\
\dot{X}_{4} \\
\dot{X}_{5}
\end{array}\right]=}\left(\omega(\pi) \mathbf{C}(v, \eta)-(g+\mu) \mathbf{I}_{3}\right)\left[\begin{array}{c}
X_{3} \\
X_{4} \\
X_{5}
\end{array}\right]-\mathbf{e}_{1} \\
&+\mathbf{L}\left(x_{1}, x_{2}, v, \eta, \omega(\pi)\right)\left[\begin{array}{c}
X_{3} \\
X_{4} \\
X_{5}
\end{array}\right] \\
&-l\left(-\frac{1}{\sigma} x_{1}\right) \mathbf{e}_{1}-\frac{\omega(\pi)}{\sigma} h\left(x_{1}, x_{2}, v, \eta\right)\left[\begin{array}{c}
X_{3} \\
X_{4} \\
X_{5}
\end{array}\right]
\end{aligned}
$$




$$
\dot{v}=0 \text {, }
$$

where $\left(x_{1}, x_{2}, X_{3}, X_{4}, X_{5}, v\right) \in \mathbb{R}^{2} \times \mathbb{R}_{++}^{3} \times\left(-v_{1}(\eta), v_{2}(\eta)\right)$. The right hand side of the system of differential equations composed of (25), (26) and (27) is at least twice continuously differentiable relative to $\left(x_{1}, x_{2}, X_{3}, X_{4}, X_{5}, v\right) \in \mathbb{R}^{2} \times \mathbb{R}_{++}^{3} \times\left(-v_{1}(\eta), v_{2}(\eta)\right){ }^{6}\left(x_{1}, x_{2}, X_{3}, X_{4}, X_{5}, v\right)=\left(0,0, \mathbf{X}^{*}(\nu, \theta), 0\right)$ is a steady state of this system. Characteristic roots of the system at this steady state are given by those of $\mathbf{J}(0, \eta, \omega(\pi))$, those of $\omega(\pi) \mathbf{C}(0, \eta)-(g+\mu) \mathbf{I}_{3}$, and 0 , where $\mathbf{J}(\nu, \eta, \omega)$ is a $2 \times 2$ matrix given by the definition (19). Since $\mathbf{J}(0, \eta, \omega(\pi))$ has two center roots as a characteristic root, and since $\omega(\pi) \mathbf{C}(0, \eta)-(g+\mu) \mathbf{I}_{3}$ has three unstable roots as a characteristic root by Lemma 2, the system composed of (25) and (27) constitutes a bifurcation diagram. ${ }^{7}$

Let $\hat{\mu}(\theta): \mathbb{R}^{2} \times \mathbb{R}_{++}^{3} \times\left(-v_{1}(\eta), v_{2}(\eta)\right) \rightarrow \mathbb{R}$ be a continuos function of $\left(x_{1}, x_{2}, X_{3}, X_{4}, X_{5}, \nu\right)$ defined as

$$
\begin{aligned}
& \hat{\mu}\left(x_{1}, x_{2}, X_{3}, X_{4}, X_{5}, v, \theta\right):= \\
& \mathbf{e}_{3}^{\mathrm{T}} \omega(\pi) \mathbf{C}(v, \eta)\left[\begin{array}{c}
\frac{X_{3}}{X_{5}} \\
\frac{X_{4}}{X_{5}} \\
1
\end{array}\right]-g+\mathbf{e}_{3}^{\mathrm{T}} \mathbf{L}\left(x_{1}, x_{2}, v, \eta, \omega(\pi)\right)\left[\begin{array}{c}
\frac{X_{3}}{X_{5}} \\
\frac{X_{4}}{X_{5}} \\
1
\end{array}\right] .
\end{aligned}
$$

For $v \in\left(-v_{1}(\eta), v_{2}(\eta)\right)$, we obtain from the equation (16)

$$
\hat{\mu}\left(0,0, \mathbf{X}^{*}(\nu, \theta), v, \theta\right)=\mu
$$

with $\rho-(1-\sigma) \mu>0$.

Let $\mathbf{H}\left(P_{1}, P_{2}, P_{3}, v, \theta\right)$ be a $3 \times 3$ matrix-valued function of $\left(P_{1}, P_{2}, P_{3}, v\right) \in \mathbb{R}_{++}^{3} \times\left(-v_{1}(\eta), v_{2}(\eta)\right)$ defined as

$$
\mathbf{H}\left(P_{1}, P_{2}, P_{3}, v, \theta\right):=\omega(\pi) \mathbf{C}(\nu, \eta)+\mathbf{L}\left(\log \frac{P_{1}}{P_{3}}, \log \frac{P_{2}}{P_{3}}, v, \eta, \omega(\pi)\right) .
$$

Let $N(\nu, \theta)$ be a set defined as $N(\nu, \theta):=\left\{(\mathbf{K}, \mathbf{P}) \in \mathbb{R}_{++}^{6}: \mathbf{H}(\mathbf{P}, v, \theta) \mathbf{K}^{\mathrm{T}}>\mathbf{0}_{3}\right\}$.

Let $N_{1}(\nu, \theta)$ be a set defined as

$$
N_{1}(\nu, \theta):=\left\{\left(\mathbf{x}_{1}, \mathbf{X}_{2}\right) \in \mathbb{R}^{2} \times \mathbb{R}_{++}^{3}:\left[\omega(\pi) \mathbf{C}(\nu, \eta)+L\left(\mathbf{x}_{1}, \nu, \eta, \omega(\pi)\right)\right] \mathbf{X}_{2}^{\mathrm{T}}>\mathbf{0}_{3}\right\} .
$$

By construction for $v \in\left(-v_{1}(\eta), v_{2}(\eta)\right)$,

$$
\left(0,0, \mathbf{X}^{*}(\nu, \theta)\right) \in N_{1}(\nu, \theta),
$$

and also by construction for $v \in\left(-v_{1}(\eta), v_{2}(\eta)\right),(\mathbf{K}, \mathbf{P}) \in N(\nu, \theta)$, if and only if $\left(x_{1}, x_{2}, X_{3}, X_{4}, X_{5}\right) \in N_{1}(\nu, \theta) \wedge$ $P_{3}>0$.

Let $a=a(\eta, \omega)$ be a number defined by the formula (42) in Appendix 4. Let $\Theta_{1}$ be a set defined as

$$
\Theta_{1}:=\{(\eta, \pi) \in \Theta: a(\eta, \omega(\pi))<0\} .
$$

As shown by Example 4 to Example $7, \Theta_{1}$ is non-empty. For $\varepsilon>0$, let $U(0,0, \varepsilon) \subset \mathbb{R}^{2}$ be defined as

$$
U(0,0, \varepsilon):=\left\{(x, y) \in \mathbb{R}^{2}: x^{2}+y^{2}<\varepsilon\right\} .
$$

Then the following proposition holds by the center manifold theorem (Guckenheimer and Holmes [1983, Theorem 3.2.1]) and the Hopf bifurcation theorem (Guckenheimer and Holmes [1983, Theorem 3.4.2]).

\section{Proposition 3} ties.

Suppose that $\theta \in \Theta_{1}$. Then there is a set of positive constants $\left(\varepsilon_{0}(\theta), \varepsilon_{1}(\theta), \varepsilon_{2}(\theta)\right) \in \mathbb{R}_{++}^{3}$ with the following proper-

1. There is an open subset $M(\theta)$ of $U\left(0,0, \varepsilon_{0}(\theta)\right)$ such that $(0,0) \in M(\theta)$ and that $M(\theta)$ is homeomorphic to $U(0,0,1)$.

2. $0<\varepsilon_{1}(\theta) \leq \nu_{1}(\eta)$, and $0<\varepsilon_{2}(\theta) \leq v_{2}(\eta)$ 
a. Suppose that $\eta<\frac{\sqrt{2}-1}{3}$. For each $v \in\left(-\varepsilon_{1}(\theta), 0\right)$, the system (25) has a unique closed orbit in $M(\theta)$, and $M(\theta) \backslash\{(0,0)\}$ constitutes a stable manifold of this closed orbit in the system (25). For each $v \in\left[0, \varepsilon_{2}(\theta)\right)$, the system (25) has a unique stable steady state $(0,0)$ in $M(\theta)$, and $M(\theta)$ constitutes a stable manifold of this steady state.

b. Suppose that $\eta>\frac{\sqrt{2}-1}{3}$. For each $v \in\left(-\varepsilon_{1}(\theta), 0\right]$, the system (25) has a unique stable steady state $(0,0)$ in $M(\theta)$, and $M(\theta)$ constitutes a stable manifold of this steady state. For each $v \in\left(0, \varepsilon_{2}(\theta)\right)$, the system (25) has a unique closed orbit in $M(\theta)$, and $M(\theta) \backslash\{(0,0)\}$ constitutes a stable manifold of this closed orbit in the system (25).

3. For each $\theta \in \Theta_{1}$, there exists a function $\varphi(\theta)$ from $M(\theta) \times\left(-\varepsilon_{1}(\theta), \varepsilon_{2}(\theta)\right)$ to $\mathbb{R}_{++}^{3}$ with the following properties.

i. $\varphi(\theta)=\varphi(x, y, v, \theta)$ is continuously differentiable relative to $(x, y, v) \in M(\theta) \times\left(-\varepsilon_{1}(\theta), \varepsilon_{2}(\theta)\right)$.

ii. $\mathbf{X}^{*}(\nu, \theta)=\varphi(0,0, \nu, \theta)$.

iii.

$$
\left\{\left(\mathbf{x}_{1}, \mathbf{X}_{2}, v\right) \in M(\theta) \times \mathbb{R}_{++}^{3} \times\left(-\varepsilon_{1}(\theta), \varepsilon_{2}(\theta)\right): \mathbf{X}_{2}=\varphi\left(\mathbf{x}_{1}, \nu, \theta\right)\right\}
$$

constitutes a center manifold of the steady state $\left(0,0, \mathbf{X}^{*}(\nu, \theta), 0\right)$ in the system of differential equations composed of (25), (26) and (27).

iv. For each $\left(\mathbf{x}_{1}, v\right) \in M(\theta) \times\left(-\varepsilon_{1}(\theta), \varepsilon_{2}(\theta)\right)$,

$$
\rho-(1-\sigma) \hat{\mu}\left(\mathbf{x}_{1}, \varphi\left(\mathbf{x}_{1}, \nu, \theta\right), \nu, \theta\right)>0
$$

v. For each $v \in\left(-\varepsilon_{1}(\theta), \varepsilon_{2}(\theta)\right)$,

$$
\left\{\left(\mathbf{x}_{1}, \mathbf{X}_{2}\right) \in M(\theta) \times \mathbb{R}_{++}^{3}: \mathbf{X}_{2}=\varphi\left(\mathbf{x}_{1}, \nu, \theta\right)\right\} \subset N_{1}(\nu, \theta) .
$$

\subsection{Stability of closed orbit}

We have sufficient preparations to analyze the stability and the determinacy of equilibrium around a closed orbit obtained by the supercritical Hopf bifurcation. Let $\Phi_{1}, \Phi_{2}$, and $\Phi$ be defined as

$$
\begin{aligned}
\Phi_{1} & :=\left\{(\nu, \theta) \in \mathbb{R} \times \Theta_{1}: \eta<\frac{\sqrt{2}-1}{3} \wedge-\varepsilon_{1}(\theta)<\nu<0\right\} \\
\Phi_{2} & :=\left\{(\nu, \theta) \in \mathbb{R} \times \Theta_{1}: \eta>\frac{\sqrt{2}-1}{3} \wedge 0<\nu<\varepsilon_{2}(\theta)\right\} \\
\Phi & :=\Phi_{1} \cup \Phi_{2} .
\end{aligned}
$$

Then $\Phi_{1}$ is non-empty by Proposition 3 and Example 7, and $\Phi_{2}$ is also non-empty by Proposition 3 and Example 4 to Example 6. In the present subsection we suppose that $\theta \in \Theta_{1}$ and that $(\nu, \theta) \in \Phi$.

Let $V(\theta)$ be a set in $\mathbb{R}^{5}$ defined as

$$
V(\theta):=M(\theta) \times \mathbb{R}_{++}^{3} .
$$

Let $S(\nu, \theta) \subset V(\theta)$ be a two-dimensional manifold defined as

$$
S(\nu, \theta):=\left\{\left(\mathbf{x}_{1}, \mathbf{X}_{2}\right) \in M(\theta) \times \mathbb{R}_{++}^{3}: \mathbf{x}_{1} \neq(0,0) \wedge \mathbf{X}_{2}=\varphi\left(\mathbf{x}_{1}, \nu, \theta\right)\right\} .
$$

Let $F(\nu, \theta): V(\theta) \rightarrow \mathbb{R}^{5}$ be a function of $\left(\mathbf{x}_{1}, \mathbf{X}_{2}\right) \in M(\theta) \times \mathbb{R}_{++}^{3}$ given by the right hand side of the system of differential equations composed of (25) and (26). Consider the following ordinary differential equation.

$$
\left(\mathbf{x}_{1}, \dot{\mathbf{x}}_{2}\right)^{\mathrm{T}}=F\left(\mathbf{x}_{1}, \mathbf{x}_{2}, v, \theta\right)
$$


where $\left(\mathbf{x}_{1}, \mathbf{X}_{2}\right) \in M(\theta) \times \mathbb{R}_{++}^{3}$. A steady state of the system (31) is given by $\left(0,0, \mathbf{X}^{*}(\nu, \theta)\right)$. By Lemma 2 and by Proposition $3.2\left(0,0, \mathbf{X}^{*}(\nu, \theta)\right)$ is a source, and its unstable manifold includes $\{(0,0)\} \times \mathbb{R}_{++}^{3}$. By Proposition 3.2 the system (31) has a closed orbit in $S(\nu, \theta)$. We denote this closed orbit by $\gamma(\nu, \theta)$. Let $W(\gamma, \nu, \theta)$ be a set of all points in $V(\theta) \cap N_{1}(\nu, \theta) \omega$-limit points of which under the action of the differential equation $(31)$ onto $N_{1}(\nu, \theta)$ belongs to $\gamma(\nu, \theta){ }^{8}$ By Proposition 3.3.iv and $\mathrm{v}$, a solution of the ordinary differential equation (31) starting from each given point in $W(\gamma, v, \theta)$ constitutes an interior optimal solution of the intertemporal optimization problem (24). We have the following relations by construction and by Lemma 2 and Proposition 3.

$$
\begin{gathered}
S(\nu, \theta) \subset W(\gamma, \nu, \theta) \subset N_{1}(\nu, \theta) \\
W(\gamma, v, \theta) \subset(M(\theta) \backslash\{(0,0)\}) \times \mathbb{R}_{++}^{3} .
\end{gathered}
$$

Therefore by the definition (30), if $W(\gamma, v, \theta)$ is a two-dimensional manifold, then $W(\gamma, v, \theta)$ coincides with $S(\nu, \theta)$. And if $W(\gamma, \nu, \theta)=S(\nu, \theta)$, then $S(\nu, \theta)$ constitutes a local stable manifold of the closed orbit $\gamma(\nu, \theta)$.

Let $\left(z_{1}, z_{2}, q_{1}, q_{2}, q_{3}\right)$ be a set of variables given by the definition (39) in Appendix 3 . Then as discussed in Section 3.3, $z_{i}, i=1,2$, are predetermined variables, and $q_{i}, i=1,2,3$, are non-predetermined variables. Let $\mathbf{M}(\sigma)$ be the $5 \times 5$ matrix given by the definition (40) in Appendix 3. Then $\operatorname{det} \mathbf{M}(\sigma) \neq 0$, and for $\mathbf{x}_{1}=\left(x_{1}, x_{2}\right) \in$ $M(\theta)$ and $\mathbf{X}_{2}=\left(X_{3}, X_{4}, X_{5}\right) \in \mathbb{R}_{++}^{3}$, we have

$$
\begin{gathered}
\left(z_{1}, z_{2}, q_{1}, q_{2}, q_{3}\right)=\left(x_{1}, x_{2}, \log X_{3}, \log X_{4}, \log X_{5}\right) \mathbf{M}(\sigma)^{\mathrm{T}} \\
\left(x_{1}, x_{2}, \log X_{3}, \log X_{4}, \log X_{5}\right)=\left(z_{1}, z_{2}, q_{1}, q_{2}, q_{3}\right)\left(\mathbf{M}(\sigma)^{-1}\right)^{\mathrm{T}} .
\end{gathered}
$$

Let $W(\bar{x}, \gamma, \nu, \theta)$ be a set defined as $W(\bar{x}, \gamma, \nu, \theta):=\left\{\left(x_{1}, x_{2}, x_{3}, x_{4}, x_{5}\right) \in M(\theta) \times \mathbb{R}^{3}:\left(x_{1}, x_{2}, e^{x_{3}}, e^{x_{4}}, e^{x_{5}}\right) \in\right.$ $W(\gamma, v, \theta)\}$.

Let $N_{2}(\nu, \theta)$ be a set defined as

$$
N_{2}(\nu, \theta):=\left\{\left(x_{1}, x_{2}, x_{3}, x_{4}, x_{5}\right) \in \mathbb{R}^{5}:\left(x_{1}, x_{2}, e^{x_{3}}, e^{x_{4}}, e^{x_{5}}\right) \in N_{1}(\nu, \theta)\right\} .
$$

By construction $W(\bar{x}, \gamma, \nu, \theta) \subset N_{2}(\nu, \theta)$. Let $W(\bar{z}, \bar{q}, \gamma, \nu, \theta)$ be a set defined as

$$
W(\bar{z}, \bar{q}, \gamma, \nu, \theta):=\left\{(\mathbf{z}, \mathbf{q}) \in \mathbb{R}^{2} \times \mathbb{R}^{3}:(\mathbf{z}, \mathbf{q})\left(\mathbf{M}(\sigma)^{-1}\right)^{\mathrm{T}} \in W(\bar{x}, \gamma, \nu, \theta)\right\}
$$

Then by construction $W(\bar{z}, \bar{q}, \gamma, v, \theta)$ one to one corresponds to $W(\gamma, v, \theta)$ under the coordinate transformation (32). Let $\operatorname{Pr}: \mathbb{R}^{2} \times \mathbb{R}^{3} \rightarrow \mathbb{R}^{2}$ be a projection operator defined as

$$
\operatorname{Pr}(\mathbf{z}, \mathbf{q})=\mathbf{z}
$$

for $(\mathbf{z}, \mathbf{q}) \in \mathbb{R}^{2} \times \mathbb{R}^{3}$. Let $W(\bar{z}, \gamma, \nu, \theta)$ be a set defined as

$$
W(\bar{z}, \gamma, \nu, \theta):=\operatorname{Pr}(W(\bar{z}, \bar{q}, \gamma, \nu, \theta)) .
$$

In the optimal growth model (24) the preference is strictly concave and the technology is convex. Hence if the optimization problem (24) has an interior solution for a given value of initial endowment, then the optimal solution is unique for this value of initial endowment, and we infer from this convex structure that any given value of the predetermined variables $\mathbf{z} \in W(\bar{z}, \gamma, v, \theta)$ uniquely corresponds to a value of the non-predetermined valuables $\mathbf{q} \in \mathbb{R}^{3}$ in such a way that $(\mathbf{z}, \mathbf{q}) \in W(\bar{z}, \bar{q}, \gamma, v, \theta)$. In fact we can show the following lemma by applying the theorem due to Benveniste and Scheinkman (1979) to the optimal growth model (24). See Nishimura and Shigoka (2019) for the proof.

\section{Lemma 3}

Suppose that $(\nu, \theta) \in \Phi$. There exists a continuous function $\psi(\nu, \theta)$ from $W(\bar{z}, \gamma, \nu, \theta)$ to $\mathbb{R}^{3}$ such that

$$
\left\{(\mathbf{z}, \mathbf{q}) \in W(\bar{z}, \gamma, \nu, \theta) \times \mathbb{R}^{3}: \mathbf{q}=\psi(\mathbf{z}, \nu, \theta)\right\}=W(\bar{z}, \bar{q}, \gamma, \nu, \theta)
$$

Lemma 3 asserts that the graph of the continuous function $\psi(\nu, \theta)$ from $\mathbf{z} \in W(\bar{z}, \gamma, \nu, \theta)$ to $\mathbf{q} \in \mathbb{R}^{3}$ coincides with $W(\bar{z}, \bar{q}, \gamma, \nu, \theta)$. Let $S(\bar{x}, \gamma, \nu, \theta)$ be a set defined as $S(\bar{x}, \gamma, v, \theta):=\left\{\left(x_{1}, x_{2}, x_{3}, x_{4}, x_{5}\right) \in M(\theta) \times \mathbb{R}^{3}:\right.$ $\left.\left(x_{1}, x_{2}, e^{x_{3}}, e^{x_{4}}, e^{x_{5}}\right) \in S(\gamma, v, \theta)\right\}$. 
Let $S(\bar{z}, \bar{q}, v, \theta)$ be a set defined as

$$
S(\bar{z}, \bar{q}, \gamma, \nu, \theta):=\left\{(\mathbf{z}, \mathbf{q}) \in \mathbb{R}^{2} \times \mathbb{R}^{3}:(\mathbf{z}, \mathbf{q})\left(\mathbf{M}(\sigma)^{-1}\right)^{\mathrm{T}} \in S(\bar{x}, \nu, \theta)\right\}
$$

Then by construction $S(\bar{z}, \bar{q}, \gamma, v, \theta)$ is a two-dimensional manifold and

$$
S(\bar{z}, \bar{q}, \gamma, \nu, \theta) \subset W(\bar{z}, \bar{q}, \gamma, \nu, \theta)
$$

On the other hand by Lemma $3 W(\bar{z}, \bar{q}, \gamma, v, \theta)$ is included in an at most two-dimensional manifold. Therefore $W(\bar{z}, \bar{q}, \gamma, \nu, \theta)$ is in itself a two-dimensional manifold. Hence by construction $W(\bar{z}, \gamma, \nu, \theta)$ and $W(\gamma, \nu, \theta)$ are also two-dimensional manifolds. As mentioned above this implies that $S(\nu, \theta)$ constitutes a local stable manifold of $\gamma(\nu, \theta)$. Let $\left(\mathbf{z}^{*}(\nu, \theta), \mathbf{q}^{*}(\nu, \theta)\right)$ be the point that corresponds to $\left(0,0, \mathbf{X}^{*}(\nu, \theta)\right)$ under the coordinate transformation (32). Then there exists a unique equilibrium for any given value of predetermined variables in the two-dimensional manifold $\left\{\mathbf{z}^{*}(\nu, \theta)\right\} \cup W(\bar{z}, \gamma, \nu, \theta)$. Therefore the following result holds.

\section{Proposition 4}

Suppose that $(\nu, \theta) \in \Phi$. The system (31) has a closed orbit $\gamma(\nu, \theta)$. The two-dimensional manifold (30) constitutes a local stable manifold of $\gamma(\nu, \theta)$. There exists a unique equilibrium for any given value of predetermined variables in the two-dimensional manifold $\left\{\mathbf{z}^{*}(\nu, \theta)\right\} \cup W(\bar{z}, \gamma, \nu, \theta)$.

\section{Acknowledgement}

We are deeply grateful to an anonymous referee and Makoto Yano for their invaluable advice. Also we have benefitted from discussions with Shintaro Asaoka, Giovanni Bella and Luis Bettencourt.

\section{Funding}

This work was supported by the Japan Society for Promotion of Science, Grants-in-Aid for Research \#15H05729, \#16H0233598 and for Specially Promoted Research \#23000001.

\section{Appendices}

\section{Appendix 1}

Proof of Lemma 1.

By definition $\omega \mathbf{C}-(g+\mu) \mathbf{I}_{3}=\left(\omega \mathbf{I}_{3}-(g+\mu) \mathbf{B}\right) \mathbf{C}$. By construction $\mathbf{B}$ is a nonnegative matrix whose Frobenius root is equal to 1. By Assumption 1.1.b $\omega-(g+\mu)=\rho+g+\sigma \mu-(g+\mu)=\rho-(1-\sigma) \mu>0$. Thus by the theorem of Perron-Frobenius (Nikaido [1968, Theorem 7.1]) $\operatorname{det}\left(\omega \mathbf{I}_{3}-(g+\mu) \mathbf{B}\right) \neq 0$, and the inverse matrix of $\omega \mathbf{I}_{3}-(g+\mu) \mathbf{B}$ is a non-negative matrix. Since $\operatorname{det}\left(\omega \mathbf{I}_{3}-(g+\mu) \mathbf{B}\right) \neq 0, \operatorname{det}\left(\omega \mathbf{C}-(g+\mu) \mathbf{I}_{3}\right) \neq 0$ by Assumption 1.2. $\left(\omega \mathbf{C}-(g+\mu) \mathbf{I}_{3}\right)^{-1} \mathbf{e}_{1}=\left[\left(\omega \mathbf{I}_{3}-(g+\mu) \mathbf{B}\right) \mathbf{C}\right]^{-1} \mathbf{e}_{1}=\mathbf{C}^{-1}\left(\omega \mathbf{I}_{3}-(g+\mu) \mathbf{B}\right)^{-1} \mathbf{e}_{1}=\mathbf{B}\left(\omega \mathbf{I}_{3}-(g+\mu) \mathbf{B}\right)^{-1} \mathbf{e}_{1}$. Since $\mathbf{B}$ is a positive matrix, and since $\left(\omega \mathbf{I}_{3}-(g+\mu) \mathbf{B}\right)^{-1}$ is a non-negative matrix, each element of $\left(\omega \mathbf{C}-(g+\mu) \mathbf{I}_{3}\right)^{-1} \mathbf{e}_{1}$ is strictly positive.

\section{Appendix 2}

Suppose that $\operatorname{det} \mathbf{B} \neq 0$. Thus $\mathbf{C}$ does exist. Let $d_{i j s}, i, j=1,2,3, s=1,2$, be defined as 


$$
\begin{array}{cc}
d_{111}:=-\left(c_{21}+c_{31}\right), \quad d_{112}:=c_{21} \\
d_{121}:=-\left(c_{22}+c_{32}\right), \quad d_{122}:=c_{22} \\
d_{131}:=-\left(c_{23}+c_{33}\right), \quad d_{132}:=c_{23} \\
d_{211}:=c_{11}, \quad d_{212}:=-\left(c_{11}+c_{31}\right) \\
d_{221}:=c_{12}, \quad d_{222}:=-\left(c_{12}+c_{32}\right) \\
d_{231}:=c_{13}, \quad d_{232}:=-\left(c_{13}+c_{33}\right) \\
d_{311}:=c_{11}, \quad d_{312}:=c_{21} \\
d_{321}:=c_{12}, \quad d_{322}:=c_{22} \\
d_{331}:=c_{13}, \quad d_{332}:=c_{23} .
\end{array}
$$

Let $l=l(x)$ be a function of $x \in \mathbb{R}$ defined as

$$
l(x):=\sum_{n=1}^{\infty} \frac{1}{n !} x^{n} .
$$

Note that $l=l(x)$ is an analytical function and that $l(0)=0$. Let $\mathbf{L}=\mathbf{L}(x, y)$ be a $3 \times 3$ matrix-valued function of $(x, y) \in \mathbb{R}^{2}$ defied as

$$
\mathbf{L}(x, y):=\omega\left[\begin{array}{lll}
c_{11} l\left(d_{111} x+d_{112} y\right) & c_{12} l\left(d_{121} x+d_{122} y\right) & c_{13} l\left(d_{131} x+d_{132} y\right) \\
c_{21} l\left(d_{21} x+d_{212} y\right) & c_{22} l\left(d_{221} x+d_{222} y\right) & c_{23} l\left(d_{231} x+d_{232} y\right) \\
c_{31} l\left(d_{31} x+d_{312} y\right) & c_{32} l\left(d_{321} x+d_{322} y\right) & c_{33} l\left(d_{331} x+d_{332} y\right)
\end{array}\right]
$$

Note that each element of $\mathbf{L}=\mathbf{L}(x, y)$ is countably many differentiable relative to $(x, y) \in \mathbb{R}^{2}$ and that $\mathbf{L}(0,0)=$ $\mathbf{O}_{3}$, where $\mathbf{O}_{3}$ is the $3 \times 3$ zero-matrix. Let $h=h(x, y)$ be a function of $(x, y) \in \mathbb{R}^{2}$ defined as

$$
h(x, y):=l\left(c_{13} x+c_{23} y\right) .
$$

Note that $h=h(x, y)$ is countably many differentiable relative to $(x, y) \in \mathbb{R}^{2}$ and that $h(0,0)=0$.

By construction we have the following relation.

$$
\begin{aligned}
\mathbf{H}\left(P_{1}, P_{2}, P_{3}\right) & =\omega \mathbf{C}+\mathbf{L}\left(\log \frac{P_{1}}{P_{3}}, \log \frac{P_{2}}{P_{3}}\right) \\
P_{1}^{-\frac{1}{\sigma}} P_{3}^{\frac{1}{\sigma}} & =1+l\left(-\frac{1}{\sigma} \log \frac{P_{1}}{P_{3}}\right) \\
P_{1}^{C_{13}} P_{2}^{C_{23}} P_{3}^{C_{33}-1} & =1+h\left(\log \frac{P_{1}}{P_{3}}, \log \frac{P_{2}}{P_{3}}\right) .
\end{aligned}
$$

\section{Appendix 3}

Let $\mathbf{T}(\sigma)$ be a $5 \times 6$ matrix defined as

$$
\mathbf{T}(\sigma):=\left[\begin{array}{cccccc}
0 & 0 & 0 & 1 & 0 & -1 \\
0 & 0 & 0 & 0 & 1 & -1 \\
1 & 0 & 0 & 0 & 0 & \frac{1}{\sigma} \\
0 & 1 & 0 & 0 & 0 & \frac{1}{\sigma} \\
0 & 0 & 1 & 0 & 0 & \frac{1}{\sigma}
\end{array}\right] .
$$

By construction the rank of $\mathbf{T}(\sigma)$ is five, and we have 


$$
\mathbf{T}(\sigma)\left[\begin{array}{l}
k_{1} \\
k_{2} \\
k_{3} \\
p_{1} \\
p_{2} \\
p_{3}
\end{array}\right]=\left[\begin{array}{l}
x_{1} \\
x_{2} \\
x_{3} \\
x_{4} \\
x_{5}
\end{array}\right]
$$

Let $Z_{i}, i=1,2$, and $Q_{i}, i=1,2,3$ be defined as

$$
Z_{1}:=\frac{K_{1}}{K_{3}}, Z_{2}:=\frac{K_{2}}{K_{3}}, Q_{1}:=P_{1} K_{1}^{\sigma}, Q_{2}:=P_{2} K_{2}^{\sigma}, Q_{3}:=P_{3} K_{3}^{\sigma} .
$$

Let $\left(z_{1}, z_{2}, q_{1}, q_{2}, q_{3}\right)$ be defined as

$$
\left(z_{1}, z_{2}, q_{1}, q_{2}, q_{3}\right):=\left(\log Z_{1}, \log Z_{2}, \log Q_{1}, \log Q_{2}, \log Q_{3}\right)
$$

Let $\mathbf{M}(\sigma)$ be a $5 \times 5$ matrix defined as

$$
\mathbf{M}(\sigma):=\left[\begin{array}{ccccc}
0 & 0 & 1 & 0 & -1 \\
0 & 0 & 0 & 1 & -1 \\
1 & 0 & \sigma & 0 & 0 \\
0 & 1 & 0 & \sigma & 0 \\
0 & 0 & 0 & 0 & \sigma
\end{array}\right]
$$

Then $\operatorname{det} \mathbf{M}(\sigma) \neq 0$, and its inverse $\mathbf{M}(\sigma)^{-1}$ is given by

$$
\mathbf{M}(\sigma)^{-1}=\left[\begin{array}{ccccc}
-\sigma & 0 & 1 & 0 & -1 \\
0 & -\sigma & 0 & 1 & -1 \\
1 & 0 & 0 & 0 & \frac{1}{\sigma} \\
0 & 1 & 0 & 0 & \frac{1}{\sigma} \\
0 & 0 & 0 & 0 & \frac{1}{\sigma}
\end{array}\right] .
$$

Then by construction we have the following relation.

$$
\left[\begin{array}{l}
z_{1} \\
z_{2} \\
q_{1} \\
q_{2} \\
q_{3}
\end{array}\right]=\mathbf{M}(\sigma)\left[\begin{array}{l}
x_{1} \\
x_{2} \\
x_{3} \\
x_{4} \\
x_{5}
\end{array}\right],\left[\begin{array}{l}
x_{1} \\
x_{2} \\
x_{3} \\
x_{4} \\
x_{5}
\end{array}\right]=\mathbf{M}(\sigma)^{-1}\left[\begin{array}{l}
z_{1} \\
z_{2} \\
q_{1} \\
q_{2} \\
q_{3}
\end{array}\right]
$$

\section{Appendix 4}

Let $\bar{c}_{i j}=\bar{c}_{i j}(\eta)$ be the $(i, j)$-element of the inverse matrix of $\overline{\mathbf{B}}(\eta)$. Let $a=a(\eta, \omega)$ be a number defined as 


$$
\begin{aligned}
a:= & \frac{\omega}{16}\left[\bar{c}_{13}^{3}+\left(\bar{c}_{21}+\bar{c}_{31}\right)^{3}+\bar{c}_{13} \bar{c}_{23}^{2}+\bar{c}_{21}^{2}\left(\bar{c}_{21}+\bar{c}_{31}\right)\right] \\
& +\frac{\omega}{16}\left[\bar{c}_{13}^{2} \bar{c}_{23}+\bar{c}_{12}^{2}\left(\bar{c}_{12}+\bar{c}_{32}\right)+\bar{c}_{23}^{3}+\left(\bar{c}_{12}+\bar{c}_{32}\right)^{3}\right] \\
& +\frac{\omega}{16(2+3 \eta)}\left[\left(\bar{c}_{13} \bar{c}_{23}+\bar{c}_{21}\left(\bar{c}_{21}+\bar{c}_{31}\right)\right)\left(\bar{c}_{13}^{2}-\left(\bar{c}_{21}+\bar{c}_{31}\right)^{2}+\bar{c}_{23}^{2}-\bar{c}_{21}^{2}\right)\right] \\
& -\frac{\omega}{16(2+3 \eta)}\left[\left(\bar{c}_{13} \bar{c}_{23}+\bar{c}_{12}\left(\bar{c}_{12}+\bar{c}_{32}\right)\right)\left(\bar{c}_{13}^{2}-\bar{c}_{12}^{2}+\bar{c}_{23}^{2}-\left(\bar{c}_{12}+\bar{c}_{32}\right)^{2}\right)\right] \\
& -\frac{\omega}{16(2+3 \eta)}\left[\left(\bar{c}_{13}^{2}-\left(\bar{c}_{21}+\bar{c}_{31}\right)^{2}\right)\left(\bar{c}_{13}^{2}-\bar{c}_{12}^{2}\right)\right] \\
& +\frac{\omega}{16(2+3 \eta)}\left[\left(\bar{c}_{23}^{2}-\bar{c}_{21}^{2}\right)\left(\bar{c}_{23}^{2}-\left(\bar{c}_{12}+\bar{c}_{32}\right)^{2}\right)\right] .
\end{aligned}
$$

\section{Appendix 5}

Let $\bar{c}_{i j}=\bar{c}_{i j}(\eta)$ be the $(i, j)$-element of the inverse matrix of $\overline{\mathbf{B}}(\eta)$. Let $f(\eta, \omega)=f(x, y, \eta, \omega)$, and $g(\eta, \omega)=$ $g(x, y, \eta, \omega)$ be functions of $(x, y) \in \mathbb{R}^{2}$ defined as

$$
\begin{aligned}
f(x, y, \eta, \omega):= & \omega\left(e^{\bar{c}_{13}(\eta) x+\bar{c}_{23}(\eta) y}-e^{-\left(\bar{c}_{21}(\eta)+\bar{c}_{31}(\eta)\right) x+\bar{c}_{21}(\eta) y}\right) \\
& -\omega\left[\left(\bar{c}_{13}(\eta)+\bar{c}_{21}(\eta)+\bar{c}_{31}(\eta)\right) x-\left(\bar{c}_{21}(\eta)-\bar{c}_{23}(\eta)\right) y\right] \\
g(x, y, \eta, \omega):= & \omega\left(e^{\bar{c}_{13}(\eta) x+\bar{c}_{23}(\eta) y}-e^{\bar{c}_{12}(\eta) x-\left(\bar{c}_{12}(\eta)+\bar{c}_{32}(\eta)\right) y}\right) \\
& -\omega\left[\left(\bar{c}_{13}(\eta)-\bar{c}_{12}(\eta)\right) x+\left(\bar{c}_{23}(\eta)+\bar{c}_{12}(\eta)+\bar{c}_{32}(\eta)\right) y\right] .
\end{aligned}
$$

Then $f(0,0, \eta, \omega)=g(0,0, \eta, \omega)=0$ and $D f(0,0, \eta, \omega)=D g(0,0, \eta, \omega)=(0,0)$, where $D f$ and $D g$ denote the derivatives of $f$ and $g$ relative to $(x, y)$, respectively. By the definition (21) we have

$$
\left[\begin{array}{l}
f^{1}(x, y, 0, \eta, \omega) \\
f^{2}(x, y, 0, \eta, \omega)
\end{array}\right]=\left[\begin{array}{cc}
0 & -(2+3 \eta) \omega \\
(2+3 \eta) \omega & 0
\end{array}\right]\left[\begin{array}{l}
x \\
y
\end{array}\right]+\left[\begin{array}{l}
f(x, y, \eta, \omega) \\
g(x, y, \eta, \omega)
\end{array}\right]
$$

Let $\hat{a}=\hat{a}(\eta, \omega)$ be a number defined as

$$
\begin{aligned}
\hat{a}:= & \frac{1}{16}\left[f_{x x x}+f_{x y y}+g_{x x y}+g_{y y y}\right] \\
& +\frac{1}{16(2+3 \eta) \omega}\left[f_{x y}\left(f_{x x}+f_{y y}\right)-g_{x y}\left(g_{x x}+g_{y y}\right)-f_{x x} g_{x x}+f_{y y} g_{y y}\right),
\end{aligned}
$$

where $f_{x y}$ denotes $\left(\partial^{2} f / \partial x \partial y\right)(0,0, \eta, \omega)$, etc. See Guckenheimer and Holmes (1983, pp. 152-153).

\section{Appendix 6}

Proof of Lemma 2.

Note that 1 is the Frobenius root of the positive matrix $\overline{\mathbf{B}}(\eta)$. Let $\lambda_{1}(\mathbf{B})$ and $\lambda_{2}(\mathbf{B})$ be characteristic roots of $\overline{\mathbf{B}}(\eta)$ other than 1 . Then we have $1+\lambda_{1}(\mathbf{B})+\lambda_{2}(\mathbf{B})=\operatorname{tr} \overline{\mathbf{B}}(\eta)$ and $\lambda_{1}(\mathbf{B}) \times \lambda_{2}(\mathbf{B})=\operatorname{det} \overline{\mathbf{B}}(\eta)$. Since $\operatorname{tr} \overline{\mathbf{B}}(\eta)-1=$ $\frac{2}{5+12 \eta+9 \eta^{2}}$, and since $\operatorname{det} \overline{\mathbf{B}}(\eta)=\frac{1}{5+12 \eta+9 \eta^{2}}, \lambda_{1}(\mathbf{B})$ and $\lambda_{2}(\mathbf{B})$ are solutions of the following quadratic equation.

$$
x^{2}-\frac{2}{5+12 \eta+9 \eta^{2}} x+\frac{1}{5+12 \eta+9 \eta^{2}}=0 .
$$

The solutions of this equation are given by $x=\frac{1}{5+12 \eta+9 \eta^{2}}\left(1 \pm i \sqrt{4+12 \eta+9 \eta^{2}}\right)$, where $i$ is the imaginary unit. The characteristic roots of $\mathbf{C}(0, \eta)=\overline{\mathbf{B}}(\eta)^{-1}$ are the inverses of $1, \lambda_{1}(\mathbf{B})$, and $\lambda_{2}(\mathbf{B})$. Thus 1 and $1 \pm i \sqrt{4+12 \eta+9 \eta^{2}}$ 
are characteristic roots of $\mathbf{C}(0, \eta)$. Since $\omega(\pi)-(g+\mu)=(\rho+g+\sigma \mu)-(g+\mu)=\rho-(1-\sigma) \mu$, this implies that characteristic roots of $\omega(\pi) \mathbf{C}(0, \eta)-(g+\mu) \mathbf{I}_{3}$ are given by $\rho-(1-\sigma) \mu$ and $\rho-(1-\sigma) \mu \pm i \omega(\pi) \sqrt{4+12 \eta+9 \eta^{2}}$. The real parts of characteristic roots of $\omega(\pi) \mathbf{C}(0, \eta)-(g+\mu) \mathbf{I}_{3}$ are all equal to $\rho-(1-\sigma) \mu$. Since $\pi \in \Pi$, $\rho-(1-\sigma) \mu>0$.

\section{Notes}

1 Caballé and Santos (1993) treat the case where a hyperbolic steady state has two unstable and one stable roots.

2 Benhabib and Nishimura (1979) has pursued this line of research in continuous time and multisector models of optimal bounded growth. 3 See Chamley (1993), Benhabib and Perli (1994), Benhabib, Perli, and Xie (1994), Benhabib, Meng, and Nishimura (2000), Nishimura and Shigoka (2006), Mattana, Nishimura, and Shigoka (2009), and Brito and Venditti (2010) for the second approach.

4 We denote the transpose of a given vector $\mathbf{a}($ resp. a given matrix $\mathbf{Q})$ by $\mathbf{a}^{\mathrm{T}}\left(\mathrm{resp} . \mathbf{Q}^{\mathrm{T}}\right.$ ).

5 As shown by Lemma 2 below, $\omega \overline{\mathbf{B}}(\eta)^{-1}-(g+\mu) \mathbf{I}_{3}$ has three unstable roots as its characteristic root.

6 For $r>1$, a center manifold of a system of differential equations given by an $r$-times continuously differentiable function is an $(r-1)$-times continuously differentiable manifold. Thus one should have $r>1$, if one would appeal to the center manifold theorem. In our case we have checked $r \geq 2$.

7 In the previous subsection we have applied the Hopf bifurcation theorem to this diagram.

8 See Hirsch and Smale (1974) for the concept of an $\omega$-limit point.

\section{References}

Benhabib, J., Q. Meng, and K. Nishimura. 2000. “Indeterminacy Under Constant Returns to Scale in Multisector Economies." Econometrica 68: 1541-1548.

Benhabib, J., and K. Nishimura. 1979. "The Hopf Bifurcation and the Existence and Stability of Closed Orbits in Multisector Models of Optimal Economic Growth." Journal of Economic Theory 21: 421-444.

Benhabib, J., and R. Perli. 1994. "Uniqueness and Indeterminacy: On the Dynamics of Endogenous Crowth." Journal of Economic Theory 63: 113-142.

Benhabib, J., R. Perli, and D. Xie. 1994. “Monopolistic Competition, Indeterminacy, and Crowth." Ricerche Economiche 48: 279-298.

Benveniste, L. M., and J. A. Scheinkman. 1979. "On the Differentiability of the Value Function in Dynamic Models of Economics." Econometrica 47: 727-732.

Brito, P., and A. Venditti. 2010. "Local and Clobal Indeterminacy in Two-Sector Models of Endogenous Growth." Journal of Mathematical Economics 46: 893-911.

Caballé, J., and M. Santos. 1993. “On Endogenous Growth with Physical and Human Capital." Journal of Political Economy 101: $1042-1067$.

Chamley, C. 1993. "Externalities and Dynamics in Models of 'Learning or Doing'." International Economic Review 34: 583-609.

Guckenheimer, J., and P. Holmes. 1983. Nonlinear Oscillations, Dynamical Systems, and Bifurcations of Vector Fields. New York: Springer-Verlag.

Hirsch, M. W., and S. Smale. 1974. Differential Equations, Dynamical Systems, and Linear Algebra. New York: Academic Press.

Kamien, M. I., and N. L. Schwartz. 1991. Dynamic Optimization. 2nd edn. New York: North-Holland.

Mattana, P., K. Nishimura, and T. Shigoka. 2009. "Homoclinic Bifurcation and Clobal Indeterminacy of Equilibrium in a Two-Sector Endogenous Growth Model." International Journal of Economic Theory 5: 25-47.

Nikaido, H. 1968. Convex Structures and Economic Theory. New York: Academic Press.

Nishimura, K., and T. Shigoka. 2006. "Sunspots and Hopf Bifurcations in Continuous Time Endogenous Crowth Models." International Journal of Economic Theory 2: 199-216.

Nishimura, K., and T. Shigoka. 2019. “Technical Appendix: Proof of Lemma 3.” mimeo.

Uzawa, H. 1965. “Optimal Technical Change in an Aggregate Model of Economic Growth.” International Economic Review 6: 18-31.

Supplementary Material: The online version of this article offers supplementary material (DOI: https://doi.org/10.1515/snde-2019-0017). 